\title{
OPTIMAL CONTROL OF A STOCHASTIC PROCESSING SYSTEM DRIVEN BY A FRACTIONAL BROWNIAN MOTION INPUT
}

\author{
ARKA P. GHOSH, ${ }^{*}$ \\ ALEXANDER ROITERSHTEIN ${ }^{* *}$ AND \\ ANANDA WEERASINGHE, ${ }^{* * *}$ Iowa State University
}

\begin{abstract}
We consider a stochastic control model driven by a fractional Brownian motion. This model is a formal approximation to a queueing network with an ON-OFF input process. We study stochastic control problems associated with the long-run average cost, the infinite-horizon discounted cost, and the finite-horizon cost. In addition, we find a solution to a constrained minimization problem as an application of our solution to the long-run average cost problem. We also establish Abelian limit relationships among the value functions of the above control problems.
\end{abstract}

Keywords: Stochastic control; controlled queueing network; heavy traffic analysis; fractional Brownian motion; self-similarity; long-range dependence

2000 Mathematics Subject Classification: Primary 60K25; 68M20; 90B22

Secondary 90B 18

\section{Introduction}

Self-similarity and long-range dependence of the underlying data are two important features observed in the statistical analysis of high-speed communication networks in heavy traffic, such as local area networks (LAN) (see, for instance, [10], [22], [26], [27], [31], [36], [35], and the references therein). In theoretical models such traffic behavior has been successfully described by stochastic models associated with fractional Brownian motion (FBM) (see [14], [15], [19], [31], [33], and [34, Chapters 7.2 and 8.7]). It is well known that FBM exhibits both of these features when the associated Hurst parameter is above $\frac{1}{2}$. Therefore, understanding the behavior and control of these stochastic models is of significant interest. The non-Markovian nature of the fractional Brownian motion makes it quite difficult to study stochastic control problems for a state process driven by FBM. The techniques such as dynamic programming and analysis of the corresponding Hamilton-Jacobi-Bellmann equations which are the commonly used tools in the analysis of the stochastic control problems associated with the ordinary Brownian motion are not available for FBM models.

In this paper we study several basic stochastic control problems for a queueing model with an input described by an FBM process. Similar queueing models, but not in the context of

\footnotetext{
Received 15 August 2008; revision received 31 December 2009.

* Postal address: Department of Statistics, Iowa State University, 3216 Snedecor Hall, Ames, IA 50011, USA.

Email address: apghosh@iastate.edu

** Postal address: Department of Mathematics, Iowa State University, 420 Carver Hall, Ames, IA 50011, USA.

Email address: roiterst@iastate.edu

*** Postal address: Department of Mathematics, Iowa State University, 414 Carver Hall, Ames, IA 50011, USA.

Email address: ananda@iastate.edu
} 
control of the state process, were considered, for instance, in [20], [24], and [37]. We are aware of only a few solvable stochastic control problems in the FBM setting. Usually, the controlled state process is a solution of a linear (semilinear in [8]) stochastic differential equation driven by FBM, and the control typically affects the drift term of the stochastic differential equation. In particular, the linear-quadratic regulator control problem is addressed in [16] and [17], and a stochastic maximum principle is developed and applied to several stochastic control problems in [3]. We refer the reader to [16] and to Chapter 9 of [4] for further examples of control problems in this setting. In contrast to the models considered in the above references, the model described here is motivated by queueing applications and involves processes with state constraints. At the end of this section, we discuss an example of a queueing network which leads to our model. Our analysis relies on a coupling of the state process with its stationary version (see [20] and [37]) which enables us to address control problems in a non-Markovian setting, and our techniques are different from those employed in [3], [8], [16], and [17].

A real-valued stochastic process $W_{H}=\left(W_{H}(t)\right)_{t \geq 0}$ is called an FBM with Hurst parameter $H \in(0,1)$ if $W_{H}(0)=0$, and $W_{H}$ is a continuous zero-mean Gaussian process with stationary increments and covariance function given by

$$
\operatorname{cov}\left(W_{H}(s), W_{H}(t)\right)=\frac{1}{2}\left[t^{2 H}+s^{2 H}-|t-s|^{2 H}\right], \quad s \geq 0, t \geq 0 .
$$

The FBM is a self-similar process with index $H$, that is, the process $\left(1 / a^{H}\right)\left(W_{H}(a t)\right)_{t \geq 0}$ for any $a>0$ has the same distribution as $\left(W_{H}(t)\right)_{t \geq 0}$. If $H=\frac{1}{2}$ then $W_{H}$ is an ordinary Brownian motion, and if $H \in\left[\frac{1}{2}, 1\right)$ then the increments of the process are positively correlated and the process exhibits long-range dependence, which means that

$$
\sum_{n=1}^{\infty} \operatorname{cov}\left(W_{H}(1), W_{H}(n+1)-W_{H}(n)\right)=\infty .
$$

Note that FBM is a recurrent process with stationary increments. Hence, $\lim _{t \rightarrow \infty} W_{H}(t) / t=0$ almost surely (a.s.) and, consequently, $\lim _{t \rightarrow \infty}\left(W_{H}(t)-u t\right)=-\infty$ a.s. for any $u>0$. For additional properties and a more detailed description of this process, we refer the reader to [23], [24], [25, Chapter 25], [28, Chapter 7.2], and [29, Chapter III].

We consider a single-server stochastic processing network having deterministic service process with rate $\mu>0$. For any time $t \geq 0$, the cumulative work input to the system over the time interval $[0, t]$ is given by $\lambda t+W_{H}(t)$, where $\lambda$ is a fixed constant and $W_{H}$ is an FBM with Hurst parameter $H \in\left[\frac{1}{2}, 1\right)$. We assume that the service rate $\mu$ satisfies $\mu>\lambda$ and that the parameter $\mu$ can be controlled. The workload present in the system at time $t \geq 0$ is given by $X_{u}^{x}(t)$ which is defined in (2.1) and (2.2), below. Here $x \geq 0$ is the initial workload and $u=\mu-\lambda>0$ is the control variable. Assuming for simplicity that $x=0$, an equivalent representation for the process $X_{u}^{x}$ is given by (see (2.12), below, for the general case)

$$
X_{u}^{x}(t)=\left(W_{H}(t)-u t\right)-\inf _{s \in[0, t]}\left(W_{H}(s)-u s\right), \quad t \geq 0 .
$$

For a given arrival process $W_{H}$, this is a common formulation for a simple stochastic network where the server works continuously unless there are no customers in the system. The first term above represents the difference between the cumulative number of job arrivals and completed services in the time interval $[0, t]$, and the last term ensures that the queue length is nonnegative, and it is a nondecreasing process which increases only when the queue-length process is zero. For more examples of such formulations for queueing networks or stochastic processing 
networks, we refer the reader to [12, Chapter 3] and [34, Chapter 9.2]. The above queueing model with $W_{H}$ in (1.1) being an FBM was considered by Zeevi and Glynn [37], and we are motivated by their work.

Our goal is to address several stochastic control problems related to the control of the workload process $X_{u}^{x}$ described above. The organization of the paper is as follows. We will conclude this section with a motivating example of a queueing network which leads to our model. In Section 2 we introduce the model and describe three basic stochastic control problems associated with it, namely the long-run average cost problem, the infinite-horizon discounted cost problem, and the finite-horizon control problem. Here we also discuss some properties of the reflection map which will be used in our analysis.

In Section 3 we study the long-run average cost problem. Here we obtain an explicit deterministic representation of the cost functional for each control $u>0$. This enables us to reduce the stochastic control problem to a deterministic minimization problem. We obtain an optimal control $u^{*}>0$ and show its uniqueness under additional convexity assumptions for the associated cost functions. We show that the value function and the corresponding optimal control are independent of the initial data. It is well known that the above property is true for the classical long-run average cost problem associated with nondegenerate diffusion processes. Here we show that it remains valid for our model driven by FBM which is highly non-Markovian. The main results of this section are given in Theorems 3.1 and 3.2. Their generalizations are given in Theorems 3.3 and 3.4. Our proofs here rely on the use of a coupling of the underlying stochastic process with its stationary version introduced in [20]. In particular, we show that the coupling time has finite moments in Proposition 3.1. Because of the highly non-Markovian character of the FBM (it is well known that FBM cannot be represented as a function of a finite-dimensional Markov process), coupling arguments in general do not work for the models associated with FBM (we refer the reader to [11] for an exception). In our case, the coupling is available due to the uniqueness results related to the reflection map described in Section 2.

We use our results in Section 3 to obtain an optimal strategy for a constrained optimization problem in Theorem 4.1 of Section 4. Similar stochastic control problems for systems driven by an ordinary Brownian motion were previously considered in [1] and [32]. An interesting application of this model to wireless networks is discussed in [1]. Our constrained optimization problem (in the FBM setting) is a basic example of a general class of problems with an added bounded variation control process in the model. This class of problems has important applications to the control of queueing networks, but in the FBM setting, it seems to be an unexplored area of research.

In Section 5 we address the infinite-horizon discounted cost problem associated with a similar cost structure. An optimal control for this problem is given in Theorem 5.1.

In Section 6 we establish Abelian limit relationships among the value functions of the three stochastic control problems introduced in Section 2. The main result of this section is stated in Theorem 6.1. We show that the long-term asymptotic for the finite-horizon control problem and the asymptotic for the infinite-horizon discounted control problem, as the discount factor approaches zero, share a common limit. This limit turns out to be the value of the long-run average cost control problem. Our proof also shows that the optimal control for the discounted cost problem converges to the optimal control for the long-run average cost control problem when the discount factor approaches zero. A similar result holds also for the optimal control of the finite-horizon problem, as the time horizon tends to infinity. For a class of controlled diffusion processes, analogous results were previously obtained in [32]. 


\subsection{Motivating example}

We conclude this section with a description of a queueing network related to the Internet traffic data in which the weak limit of a suitably scaled queue-length process satisfies (2.1) and (2.2) (which are reduced to (1.1) when the initial workload $x$ equals 0 ), below. For more details on this model, we refer the reader to [31] and Chapters 7 and 8 of [34].

We begin by defining a sequence of queueing networks with state-dependent arrival and service rates, indexed by an integer $n \geq 1$ and a nonnegative real-valued parameter $\tau \geq 0$. For each $n \geq 1$ and $\tau \geq 0$, the $(n, \tau)$ th network has only one server and one buffer of infinite size, and the arrivals and departures from the system are given as follows. There are $n$ input sources (e.g. $n$ users connected to the server), and job requirements of each user are given by the so-called $O N-O F F$ process $\left\{X_{i}^{n, \tau}, i \geq 1\right\}$ as defined in [31], namely each user stays connected to the server for a random $\mathrm{ON}$ period of time with distribution function $F_{1}$, and stays off during a random OFF period of time with distribution function $F_{2}$. The distribution $F_{i}$ is assumed to have finite mean $m_{i}$ but infinite variance, and in particular,

$$
1-F_{i}(x) \sim c_{i} x^{-\alpha_{i}}
$$

where $1<\alpha_{i}<2$ and $c_{i}>0, i=1,2$, are constants. While connected to the server, each user demands service at unit rate (sends data packets at a unit rate to the server for processing). The server is processing users requests at a constant rate, say $\mu_{n, \tau}$. Assume that the $\mathrm{ON}$ and OFF periods are all independent (for each user as well as across users), the ON-OFF processes have stationary increments, and the average rate of arrival of jobs (packets) from each source (customer) is given by $\lambda=m_{1} /\left(m_{1}+m_{2}\right)$. The queue length at time $t \geq 0$ is given by

$$
X^{n, \tau}(t)=X^{n, \tau}(0)+\sum_{i=1}^{n} \int_{0}^{t} X_{i}^{n, \tau}(s) \mathrm{d} s-\mu_{n, \tau} t+L^{n, \tau}(t),
$$

where $L^{n, \tau}$ is a nondecreasing process that starts from 0 , increases only when $X^{n, \tau}$ is 0 , and ensures that $X^{n, \tau}$ is always nonnegative. Physically, this implies that the server is nonidling, i.e. it serves jobs continuously as long as the buffer is nonempty. The second term on the right-hand side of the above equation represents the cumulative number of packets sent to the server by all the $n$ customers in the interval $[0, t]$. We will assume that $X^{n, \tau}(0)=x_{n, \tau}$, where $x_{n, \tau}$ are fixed nonnegative real numbers for each $n$ and $\tau$. In this setup, $\tau>0$ represents the time scaling parameter, and it is well known (see [30] or Theorem 7.2.5 of [34]) that

$$
\tau^{-H} n^{-1 / 2} \sum_{i=1}^{n} \int_{0}^{\tau}\left(X_{i}^{n, \tau}(s)-\lambda\right) \mathrm{d} s \Rightarrow W_{H}(\cdot),
$$

when $n \rightarrow \infty$ first and then $\tau \rightarrow \infty$. Here $W_{H}$ is an FBM with Hurst parameter $H=$ $\left(3-\min \left\{\alpha_{1}, \alpha_{2}\right\}\right) / 2 \in\left(\frac{1}{2}, 1\right)$, and the convergence is the weak convergence in the space $D([0, \infty), \mathbb{R})$ of right-continuous real functions with left limits equipped with the standard $J_{\alpha, 1}$ topology (see [34, Chapter 3.3] for details).

From the above convergence result, it can be deduced (see Theorem 8.7.1 of [34]) that if the service rates $\mu_{n, \tau}$ satisfy the heavy traffic assumption

$$
\tau^{-H} n^{-1 / 2}\left(\mu_{n, \tau}-n \lambda \tau\right) \rightarrow u
$$

as $(n, \tau) \rightarrow \infty$, then a suitably scaled queue length satisfies equations (2.1) and (2.2), below. 
More precisely, if the above heavy traffic condition is satisfied, and $\tau^{-H} n^{-1 / 2} x_{n, \tau} \rightarrow x$, then the scaled queue length $\tau^{-H} n^{-1 / 2} X^{n, \tau}(\tau \cdot)$ converges weakly to a limiting process $X_{u}^{x}(\cdot)$ that satisfies (2.1) and (2.2) if we let $n \rightarrow \infty$ first and then $\tau \rightarrow \infty$. Hence, we see that, with a 'superimposed' ON-OFF input source and deterministic services times for the queueing processes, a suitably scaled queue length in the limit satisfies our model. With a cost structure similar to either (2.5), (2.7), or (2.10), below, for the queueing network problem, we can consider the problem described in this paper as a formal fractional Brownian control problem (FBCP) of the corresponding control problem for the queueing network. We do not, however, attempt to solve the queueing control problem in this paper. A solution of the limiting control problem provides useful insights into the queueing network control problem (see, for instance, [13]). For a broad class of queueing problems, it has been shown that the value function of the Brownian control problem $(\mathrm{BCP})$ is a lower bound for the minimum cost in the queueing network control problem (see [6]). In many situations, the solution to the BCP can be utilized to obtain optimal strategies for the queueing network control problem (cf. [2], [5], [9], etc.). Here, we study just the BCP, which is an important problem in its own right. Our explicit solution to the FBCP can be considered as an 'approximate solution' to the queueing network problem.

\section{Basic setup}

In this section we define the controlled state process (Section 2.1), describe three standard control problems associated with it (Section 2.2), and also discuss some basic properties of a reflection mapping which is involved in the definition of the state process (Section 2.3).

\subsection{Model}

Let $\left(W_{H}(t)\right)_{t \geq 0}$ be an FBM with Hurst parameter $H \geq \frac{1}{2}$, and let $\sigma(\cdot)$ be a deterministic continuous function defined on $[0, \infty)$ and taking positive values. For a given initial value $x \geq 0$ and a control variable $u \geq 0$, the controlled state process $X_{u}^{x}$ is defined by

$$
X_{u}^{x}(t)=x-u t+\sigma(u) W_{H}(t)+L_{u}^{x}(t), \quad t \geq 0,
$$

where the process $L_{u}^{x}$ is given by

$$
L_{u}^{x}(t)=-\min \left\{0, \min _{s \in[0, t]}\left(x-u s+\sigma(u) W_{H}(s)\right)\right\}, \quad t \geq 0 .
$$

The control variable $u \geq 0$ remains fixed throughout the evolution of the state process $X_{u}^{x}$. It follows from (2.1) and (2.2) that $X_{u}^{x}(t) \geq 0$ for all $t \geq 0$. Note that the process $L_{u}^{x}$ has continuous paths, and it increases at times when $X_{u}^{x}(t)=0$.

The process $X_{u}^{x}$ represents the workload process of a single server controlled queue fed by an FBM, as described in the previous section (see also [37]). For a chosen control $u \geq 0$ that remains fixed for all $t \geq 0$, the controller is faced with a cost structure consisting of the following three additive components during a time interval $[t, t+\mathrm{d} t]$ :

1. a control cost $h(u) \mathrm{d} t$,

2. a state-dependent holding $\operatorname{cost} C\left(X_{u}^{x}\right) \mathrm{d} t$,

3. a penalty of $p \mathrm{~d} L_{u}^{x}(t)$, if the workload in the system is empty. 
Here $p \geq 0$ is a constant, and $h$ and $C$ are nonnegative continuous functions satisfying the following basic assumptions.

(i) The function $h$ is defined on $[0,+\infty)$, and

$$
h \text { is nondecreasing and continuous, } h(0) \geq 0, \lim _{u \rightarrow+\infty} h(u)=+\infty \text {. }
$$

(ii) The function $C$ is also defined on $[0,+\infty)$, and it is a nonnegative, nondecreasing continuous function which satisfies the following polynomial growth condition:

$$
0 \leq C(x) \leq K\left(1+x^{\gamma}\right)
$$

for some positive constants $K>0$ and $\gamma>0$.

We will sometimes assume the convexity of $h$ and $C$ in order to obtain sharper results, such as the uniqueness of the optimal controls.

\subsection{Three control problems}

Here we formulate three cost minimization problems for our model. In the long-run average cost minimization problem (it is also called the ergodic control problem), the controller minimizes the cost functional

$$
\begin{aligned}
I(u, x): & =\limsup _{T \rightarrow \infty} \frac{1}{T} \mathrm{E}\left(\int_{0}^{T}\left[h(u)+C\left(X_{u}^{x}(t)\right)\right] \mathrm{d} t+\int_{0}^{T} p \mathrm{~d} L_{u}^{x}(t)\right) \\
& =h(u)+\limsup _{T \rightarrow \infty} \frac{1}{T} \mathrm{E}\left(\int_{0}^{T} C\left(X_{u}^{x}(t)\right) \mathrm{d} t+p L_{u}^{x}(T)\right),
\end{aligned}
$$

subject to the constraint $u>0$ for a fixed initial value $x \geq 0$. Here $p>0$ is a positive constant. Note that, since $L_{u}^{x}(t)$ is an increasing process, the integral with respect to $L_{u}^{x}(t)$ can be defined as an ordinary Riemann-Stieltjes integral. The value function of this problem is given by

$$
V_{0}(x)=\inf _{u>0} I(u, x) .
$$

In Section 3 we show that $I(u, x)$ and, hence, also the value function $V_{0}(x)$ are actually independent of $x$. In addition, we show the existence of a finite optimal control $u^{*}>0$ and also prove that $u^{*}$ is unique if the functions $h$ and $C$ are convex. We apply the results on the long-run average cost problem to find an optimal strategy for a constrained optimization problem in Section 4.

In Section 5 we solve the infinite-horizon discounted cost minimization problem for the case when $\sigma(u) \equiv 1$ in (2.1). In this problem it is assumed that the controller wants to minimize the cost functional

$$
J_{\alpha}(u, x):=\mathrm{E}\left(\int_{0}^{\infty} \mathrm{e}^{-\alpha t}\left[h(u)+C\left(X_{u}^{x}(t)\right)\right] \mathrm{d} t+p \int_{0}^{\infty} \mathrm{e}^{-\alpha t} \mathrm{~d} L_{u}^{x}(t)\right),
$$

subject to $u>0$ for a fixed initial value $x \geq 0$. Here the discount factor $\alpha>0$ is a positive constant. The value function in this case is given by

$$
V_{\alpha}(x)=\inf _{u>0} J_{\alpha}(u, x) .
$$

We study the asymptotic behavior of this model in Section 6. When $\alpha$ approaches 0 , we prove that $\lim _{\alpha \rightarrow 0^{+}} \alpha J_{\alpha}(u, x)=I(u, x)$ for any control $u>0$. Furthermore, we show 
that $\lim _{\alpha \rightarrow 0^{+}} \alpha V_{\alpha}(x)=V_{0}(x)$ and that the optimal controls for the discounted cost problem converge to those of the long-run average cost problem as $\alpha$ tends to 0. In Section 6 we also consider the finite-horizon control problem with the value function $V(x, T)$ defined by

$$
V(x, T):=\inf _{u>0} I(u, x, T),
$$

where

$$
\begin{aligned}
I(u, x, T): & =\mathrm{E}\left(\int_{0}^{T}\left[h(u)+C\left(X_{u}^{x}(t)\right)\right] \mathrm{d} t+p L_{u}^{x}(T)\right) \\
& =h(u) T+p \mathrm{E}\left(L_{u}^{x}(T)\right)+\mathrm{E}\left(\int_{0}^{T} C\left(X_{u}^{x}(t)\right) \mathrm{d} t\right),
\end{aligned}
$$

and $p \geq 0$ is a nonnegative constant. We also prove that $\lim _{T \rightarrow \infty} V(x, T) / T=V_{0}(x)$. Furthermore, we show that the optimal controls for the finite-horizon problem converge to that of the long-run average cost problem, as $T$ tends to $\infty$.

\subsection{The reflection map}

The model equations (2.1) and (2.2) have an equivalent representation, which is given below in (2.12) by using the reflection map. Therefore, we briefly discuss some basic properties of the reflection map and of representation (2.12).

Let $\mathcal{C}([0, \infty), \mathbb{R})$ be the space of continuous functions with domain $[0, \infty)$. The standard reflection mapping $\Gamma: \mathcal{C}([0, \infty), \mathbb{R}) \rightarrow \mathcal{C}([0, \infty), \mathbb{R})$ is defined by

$$
\Gamma(f)(t)=f(t)+\sup _{s \in[0, t]}(-f(s))^{+}
$$

for $f \in \mathcal{C}([0, \infty), \mathbb{R})$. Here and henceforth, we use the notation $a^{+}:=\max \{0, a\}$. This mapping is also known as the Skorokhod map or the regulator map in different contexts. For a detailed discussion, we refer the reader to [21] and [34, Chapter 13.5].

In our model (2.1)-(2.2) we can write $X_{u}^{x}$ as follows:

$$
X_{u}^{x}(t)=\Gamma\left(x-u e+\sigma(u) W_{H}\right)(t),
$$

where $e(t):=t$ for $t \geq 0$ is the identity map.

Note that, according to the definition, $\Gamma(f)(t) \geq 0$ for $t \geq 0$. We will also use the following two standard facts about $\Gamma$ (see, for instance, [21] and [34, Chapter 13.5]). First, we have

$$
\sup _{t \in[0, T]}|\Gamma(f)(t)| \leq 2 \sup _{t \in[0, T]}|f(t)|
$$

for $f \in \mathcal{C}([0, \infty), \mathbb{R})$. Secondly, let $f$ and $g$ be two functions in $\mathcal{C}([0, \infty), \mathbb{R})$ such that $f(0)=g(0)$ and $h(t):=f(t)-g(t)$ is a nonnegative, nondecreasing function in $\mathcal{C}([0, \infty), \mathbb{R})$. Then

$$
\Gamma(f)(t) \geq \Gamma(g)(t) \text { for all } t \geq 0 .
$$

We will also rely on the following convexity property of the reflected mapping. Let $\alpha \in(0,1)$, and let $f$ and $g$ be two functions in $\mathcal{C}([0, \infty), \mathbb{R})$. Then, $\alpha f+(1-\alpha) g \in \mathcal{C}([0, \infty), \mathbb{R})$ and

$$
\Gamma(\alpha f+(1-\alpha) g)(t) \leq \alpha \Gamma(f)(t)+(1-\alpha) \Gamma(g)(t) \quad \text { for all } t \geq 0 .
$$


The proof is straightforward. Let $F(x)=x^{-}:=\max \{0,-x\}$. Then $F$ is a convex function and, therefore,

$$
(\alpha f(s)+(1-\alpha) g(s))^{-} \leq \alpha f^{-}(s)+(1-\alpha) g^{-}(s) .
$$

Consequently,

$$
\sup _{s \in[0, t]}(\alpha f(s)+(1-\alpha) g(s))^{-} \leq \alpha \sup _{s \in[0, t]} f^{-}(s)+(1-\alpha) \sup _{s \in[0, t]} g^{-}(s) .
$$

Since

$$
\sup _{s \in[0, t]}(-f(s))^{+}=\sup _{s \in[0, t]} f^{-}(s),
$$

inequality (2.15) follows from definition (2.11).

The reflection map also satisfies the following minimality property: if $\psi, \eta \in \mathcal{C}([0, \infty), \mathbb{R})$ are such that $\psi$ is nonnegative, $\eta(0)=0, \eta$ is nondecreasing, and $\psi(t)=\varphi(t)+\eta(t)$ for $t \geq 0$, then

$$
\psi(t) \geq \Gamma(\varphi)(t) \quad \text { and } \quad \eta(t) \geq \sup _{s \in[0, t]}(-\varphi(s))^{+} \quad \text { for all } t \geq 0 .
$$

\section{Long-run average cost minimization problem}

In this section we address the control problem defined in (2.5)-(2.6). First we find a solution to the control problem for the particular case when $\sigma(u) \equiv 1$ in (2.1). This is accomplished in Sections 3.1-3.4. In Section 3.5 we show that the general case can be reduced to this simplified version.

\subsection{Reduction of the cost structure}

The controlled state space process $X_{u}^{x}$ (corresponding to $\sigma(u) \equiv 1$ ) has the form

$$
X_{u}^{x}(t)=x-u t+W_{H}(t)+L_{u}^{x}(t), \quad t \geq 0 .
$$

The following lemma simplifies the expression for the cost functional (2.5) by computing $\lim _{T \rightarrow \infty}(1 / T) \mathrm{E}\left(L_{u}^{x}(T)\right)$.

Lemma 3.1. Let $X_{u}^{x}$ be given by (3.1). Then

$$
\lim _{T \rightarrow \infty} \frac{1}{T} \mathrm{E}\left(L_{u}^{x}(T)\right)=u .
$$

Proof. Since $u>0$, using (2.12), (2.13), and (2.14), we obtain

$$
0 \leq X_{u}^{x}(t) \leq X_{0}^{x}(t)=\Gamma\left(x+W_{H}\right)(t) \leq 2\left(|x|+\sup _{s \in[0, t]}\left|W_{H}(s)\right|\right) .
$$

By the self-similarity of the FBM process,

$$
\mathrm{E}\left(\sup _{s \in[0, T]}\left|W_{H}(s)\right|\right) \leq K_{1} T^{H},
$$

where $K_{1} \in(0, \infty)$ is a constant independent of $T$ (see, for instance, [25, p. 296]). Therefore,

$$
0 \leq \mathrm{E}\left(X_{u}^{x}(T)\right) \leq 2 K_{1}\left(|x|+T^{H}\right) .
$$

Consequently, $\lim _{T \rightarrow \infty}(1 / T) \mathrm{E}\left(X_{u}^{x}(T)\right)=0$. Since $W_{H}(T)$ is a mean-zero Gaussian process, it follows from (3.1) that $(1 / T) \mathrm{E}\left(L_{u}^{x}(T)\right)-u=(1 / T)\left(\mathrm{E}\left(X_{u}^{x}(T)\right)-x\right)$. Letting $T$ tend to $\infty$ completes the proof of the lemma. 
Remark 3.1. Lemma 3.1 with literally the same proof as above remains valid if $X_{u}^{x}$ satisfies (2.1) instead of (3.1).

With the above lemma in hand, we can represent the cost functional (2.5) and reformulate the long-run average cost minimization problem as follows. The controller minimizes

$$
I(u, x)=(h(u)+p u)+\limsup _{T \rightarrow \infty} \frac{1}{T} \mathrm{E}\left(\int_{0}^{T} C\left(X_{u}^{x}(t)\right) \mathrm{d} t\right)
$$

subject to $u>0$ and $X_{u}^{x}$ given in (3.1). Note that the above reduction shows that the original minimization problem (2.5) reduces to the case $p=0$ with the function $h(u)$ replaced by $h(u)+p u$.

Our next step is to analyze the cost component $\lim _{\sup } \operatorname{sum}_{T \rightarrow \infty}(1 / T) \mathrm{E}\left(\int_{0}^{t} C\left(X_{u}^{x}(t)\right) \mathrm{d} t\right)$. The following results are described in [7] and [20], and are collected in [37] in a convenient form for our application. We summarize them here using our notation.

(i) The random sequence $X_{u}^{0}(t)$ with $t \geq 0$ converges weakly, as $t$ goes to $\infty$, to the random variable

$$
Z_{u}:=\max _{s \geq 0}\left\{W_{H}(s)-u s\right\}
$$

(ii) There is a probability space supporting the processes $X_{u}^{0}$ and $L_{u}^{0}$ (and, hence, $X_{u}^{x}$ as well as $L_{u}^{x}$ for any $\left.x \geq 0\right)$, and a stationary process $X_{u}^{*}=\left\{X_{u}^{*}(t): t \geq 0\right\}$ such that

$$
X_{u}^{*}(t)=W_{H}(t)-u t+\max \left\{X_{u}^{*}(0), L_{u}^{0}(t)\right\}, \quad t \geq 0,
$$

and

$$
X_{u}^{*}(t) \stackrel{\mathrm{D}}{=} Z_{u}, \quad t \geq 0,
$$

where ' $\stackrel{\mathrm{D}}{=}$, denotes equality in distribution and $Z_{u}$ is defined in (3.4).

(iii) The tail of the stationary distribution satisfies

$$
\lim _{\mathfrak{z} \rightarrow \infty} \mathfrak{z}^{2 H-2} \log \mathrm{P}\left(Z_{u} \geq \mathfrak{z}\right)=-\theta^{*}(u),
$$

where $\theta^{*}(u)$ is given by

$$
\theta^{*}(u)=\frac{u^{2 H}}{2 H^{2 H}(1-H)^{2(1-H)}}>0 .
$$

In particular, all the moments of $Z_{u}$ are finite.

Remark 3.2. In (ii) above, the construction of $\underset{\widetilde{W}_{u}^{*}}{*}$ is rather simple. Consider a probability space with two independent FBMs $W_{H}(\cdot)$ and $\widetilde{W}_{H}(\cdot)$. By pasting $W_{H}(\cdot)$ and $\widetilde{W}_{H}(\cdot)$ at the point $t=0$, first we construct the two-sided FBM $\left\{B_{H}(t):-\infty<t<+\infty\right\}$, which has stationary Gaussian increments. Then we define

$$
X_{u}^{*}(t)=B_{H}(t)-u t+\sup _{s \in(-\infty, t]}\left(u s-B_{H}(s)\right), \quad t \in \mathbb{R} .
$$


The random variables $X_{u}^{*}(t)$ are finite for all $t \in \mathbb{R}$ because $\lim _{|t| \rightarrow \infty} B_{H}(t) / t=0$ a.s. Note that, for all $t \in \mathbb{R}$,

$$
\begin{aligned}
X_{u}^{*}(t) & =\sup _{s \in(-\infty, t]}\left(B_{H}(t)-B_{H}(s)-u(t-s)\right) \\
& \stackrel{\mathrm{D}}{=} \sup _{s \in(-\infty, t]}\left(\widetilde{B}_{H}(t-s)-u(t-s)\right) \\
& =\sup _{r \in[0, \infty)}\left(\widetilde{B}_{H}(r)-u r\right),
\end{aligned}
$$

where $\widetilde{B}_{H}(\cdot)$ is a two-sided FBM. Hence, (3.6) holds for all $t \in \mathbb{R}$. Furthermore, $X_{u}^{*}(0)$ is independent of $\left\{W_{H}(t): t \geq 0\right\}$ and, clearly, (3.5) holds. For further discussion of (i)-(iii), we refer the reader to [7], [18], [20], and [24].

Throughout the rest of the paper, we use this probability space where all these processes are defined. Using (3.1) and (2.2), we can write, for $t \geq 0$,

$$
X_{u}^{x}(t)=W_{H}(t)-u t+\max \left\{x, L_{u}^{0}(t)\right\}
$$

where

$$
L_{u}^{0}(t)=-\inf _{s \in[0, t]}\left(W_{H}(s)-u s\right)=\sup _{s \in[0, t]}\left(u s-W_{H}(s)\right) .
$$

The above representations (3.9) and (3.10) agree with (3.5) if the process $X_{u}^{x}$ is initialized with $X_{u}^{*}(0)$.

\subsection{A coupling time}

The following coupling argument is crucial to address the optimal control problems. In particular, it enables us to deal with the last term of $I(u, x)$ in (3.3).

Proposition 3.1. Let $u>0$, and let the initial point $x \geq 0$ be fixed. Consider the state process $X_{u}^{x}$ in (3.1) and the stationary process $X_{u}^{*}$ of (3.5) and (3.6). Then the following results hold.

(i) There is a finite stopping time $\tau_{0}$ such that $X_{u}^{x}(t)=X_{u}^{*}(t)$ for all $t \geq \tau_{0}$. Furthermore, $\mathrm{E}\left(\tau_{0}^{\beta}\right)<\infty$ for all $\beta \geq 0$.

(ii) The cost functional $I(u, x)$ defined in (2.5) is finite and independent of $x$, that is, $I(u, x)=$ $I(u, 0)<\infty$ for $x \geq 0$. Consequently, the value function $V_{0}(x)=\inf _{u>0} I(u, x)$ is also finite and independent of $x$, that is $V_{0}(x)=V_{0}(0)<\infty$ for $x \geq 0$.

Proof. For $y>0$, introduce the stopping time

$$
\lambda_{y}=\inf \left\{t>0: L_{u}^{0}(t)>x+y\right\} .
$$

The stopping time $\lambda_{y}$ is finite a.s. because

$$
\lim _{t \rightarrow+\infty} L_{u}^{0}(t) \geq \lim _{t \rightarrow+\infty}\left(u t-W_{H}(t)\right)=+\infty \quad \text { a.s. }
$$

Define the stopping time $\tau_{0}$ by

$$
\tau_{0}=\inf \left\{t>0: L_{u}^{0}(t)>x+X_{u}^{*}(0)\right\} .
$$


Here $X_{u}^{*}$ is the stationary process which satisfies (3.5) and (3.6). Hence, $\tau_{0}=\lambda_{X_{u}^{*}(0)}$ a.s. It follows that, for $t \geq \tau_{0}$, we have $L_{u}^{0}(t) \geq L_{u}^{0}\left(\tau_{0}\right)=x+X_{u}^{*}(0)$ and $X_{u}^{*}(0) \geq 0$. Therefore, it follows from (3.9) and (3.10) that $X_{u}^{x}(t)=W_{H}(t)-u t+L_{u}^{0}(t)=X_{u}^{*}(t)$ for $t \geq \tau_{0}$.

Next, we prove that $\mathrm{E}\left(\tau_{0}^{\beta}\right)<+\infty$ for each $\beta \geq 0$. Without loss of generality, we can assume that $\beta \geq 1$. We then have

$$
\mathrm{E}\left(\tau_{0}^{\beta}\right) \leq \sum_{m=0}^{\infty} \mathrm{E}\left(\lambda_{m+1}^{\beta} \mathbf{1}_{\left[m \leq X_{u}^{*}(0)<m+1\right]}\right) \leq \sum_{m=0}^{\infty}\left[\mathrm{E}\left(\lambda_{m+1}^{2 \beta}\right) \mathrm{P}\left(X_{u}^{*}(0) \geq m\right)\right]^{1 / 2},
$$

where in the last step we have used the Cauchy-Schwartz inequality. Since $X_{u}^{*}(0)$ has the same distribution as $Z_{u}=\sup _{s \geq 0}\left\{W_{H}(s)-u s\right\}$, it follows from (3.7) that, for all large enough $m$,

$$
\mathrm{P}\left(X_{u}^{*}(0) \geq m\right) \leq \mathrm{e}^{-\theta^{*}(u) m^{2(1-H)} / 2},
$$

where $\theta^{*}(u)$ is defined in (3.8). Next, we estimate $\mathrm{E}\left(\lambda_{m}^{2 \beta}\right)$ for $m \geq 0$ and $\beta \geq 1$. For $m \in \mathbb{N}$, let $b_{m}=x+m$ and $T_{m}=2 b_{m} / u$. We have

$$
\begin{aligned}
\mathrm{E}\left(\lambda_{m}^{2 \beta}\right) & =2 \beta \int_{0}^{\infty} t^{2 \beta-1} \mathrm{P}\left(\lambda_{m}>t\right) \mathrm{d} t \\
& =2 \beta \int_{0}^{\infty} t^{2 \beta-1} \mathrm{P}\left(L_{u}^{0}(t) \leq x+m\right) \mathrm{d} t \\
& \leq T_{m}^{2 \beta}+2 \beta \int_{T_{m}}^{\infty} t^{2 \beta-1} \mathrm{P}\left(L_{u}^{0}(t) \leq b_{m}\right) \mathrm{d} t,
\end{aligned}
$$

where the second equality is due to the fact that $\mathrm{P}\left(\lambda_{m}>t\right)=\mathrm{P}\left(L_{u}^{0}(t) \leq x+m\right)$ according to the definition of $\lambda_{m}$. Note that

$$
\mathrm{P}\left(L_{u}^{0}(t) \leq b_{m}\right)=\mathrm{P}\left(\sup _{s \in[0, t]}\left\{u s-W_{H}(s)\right\} \leq b_{m}\right) \leq \mathrm{P}\left(W_{H}(t) \geq u t-b_{m}\right),
$$

and recall that $Z:=W_{H}(t) / t^{H}$ has a standard normal distribution. Therefore, by (3.15), for $t>T_{m}$, we have

$$
\mathrm{P}\left(L_{u}^{0}(t) \leq b_{m}\right) \leq \mathrm{P}\left(W_{H}(t) \geq u t-b_{m}\right) \leq \mathrm{P}\left(W_{H}(t) \geq \frac{u t}{2}\right)=\mathrm{P}\left(Z \geq \frac{u t^{1-H}}{2}\right) .
$$

It follows from (3.14) and (3.16) that

$$
\begin{aligned}
\mathrm{E}\left(\lambda_{m}^{2 \beta}\right) & \leq T_{m}^{2 \beta}+2 \beta \int_{0}^{\infty} t^{2 \beta-1} \mathrm{P}\left(Z \geq \frac{u t^{1-H}}{2}\right) \mathrm{d} t \\
& =T_{m}^{2 \beta}+2 \beta \int_{0}^{\infty} t^{2 \beta-1} \mathrm{P}\left(\left(\frac{2 Z}{u}\right)^{1 /(1-H)} \geq t\right) \mathrm{d} t \\
& =T_{m}^{2 \beta}+\mathrm{E}\left(\left(\frac{2|Z|}{u}\right)^{2 \beta /(1-H)}\right) \\
& =\frac{4^{\beta}}{u^{2 \beta}}(x+m)^{2 \beta}+\mathrm{E}\left(\left(\frac{2|Z|}{u}\right)^{2 \beta /(1-H)}\right) \\
& <\infty
\end{aligned}
$$


The estimates (3.13) and (3.17) imply that the infinite series on the right-hand side of (3.12) converges. Thus, $\mathrm{E}\left(\tau_{0}^{\beta}\right)<\infty$ for all $\beta \geq 1$, and, hence, for all $\beta \geq 0$. This completes the proof of the first part of the proposition.

We turn now to the proof of part (ii). First, we will prove that

$$
\mathrm{E}\left(\int_{0}^{\infty}\left|C\left(X_{u}^{x}(t)\right)-C\left(X_{u}^{*}(t)\right)\right| \mathrm{d} t\right)<\infty .
$$

We will show later that part (ii) of the proposition is a rather direct consequence of this inequality.

Note that

$$
\mathrm{E}\left(\int_{0}^{\infty}\left|C\left(X_{u}^{x}(t)\right)-C\left(X_{u}^{*}(t)\right)\right| \mathrm{d} t\right)=\mathrm{E}\left(\int_{0}^{\tau_{0}}\left|C\left(X_{u}^{x}(t)\right)-C\left(X_{u}^{*}(t)\right)\right| \mathrm{d} t\right),
$$

where $\tau_{0}$ is given in (3.11) and $X_{u}^{*}$ is given in (3.5) and (3.6). The definition of $\tau_{0}$ implies that $L_{u}^{0}\left(\tau_{0}\right) \leq x+X_{u}^{*}(0)$. Therefore, it follows from (3.4) and (3.5) that, for $t \in\left[0, \tau_{0}\right]$,

$$
\max \left\{X_{u}^{x}(t), X_{u}^{*}(t)\right\} \leq Z_{u}+x+X_{u}^{*}(0) .
$$

Since $C$ is a nondecreasing function, this implies that

$$
\max \left\{C\left(X_{u}^{x}(t)\right), C\left(X_{u}^{*}(t)\right)\right\} \leq C\left(Z_{u}+x+X_{u}^{*}(0)\right),
$$

and, consequently, using the Cauchy-Schwartz inequality,

$$
\begin{aligned}
\mathrm{E}\left(\int_{0}^{\tau_{0}}\left|C\left(X_{u}^{x}(t)\right)-C\left(X_{u}^{*}(t)\right)\right| \mathrm{d} t\right) & \leq \mathrm{E}\left(\tau_{0} C\left(Z_{u}+x+X_{u}^{*}(0)\right)\right) \\
& \leq\left[\mathrm{E}\left(\tau_{0}^{2}\right) \mathrm{E}\left(\left[C\left(Z_{u}+x+X_{u}^{*}(0)\right)\right]^{2}\right)\right]^{1 / 2} .
\end{aligned}
$$

Since $\mathrm{E}\left(\tau_{0}^{2}\right)<\infty$ by part (i) of the lemma, (3.18) will follow once we show that

$$
\mathrm{E}\left(\left[C\left(Z_{u}+x+X_{u}^{*}(0)\right)\right]^{2}\right)<\infty .
$$

Recall that $X_{u}^{*}(0)$ and $Z_{u}$ have the same distribution. The tail asymptotic (3.7) implies that any moment of $Z_{u}$ is finite. This fact together with (2.4) yields (3.19).

We will now deduce part (ii) of the proposition from (3.18). Toward this end, first observe that, since $X_{u}^{*}(t)$ is a stationary process,

$$
\frac{1}{T} \mathrm{E}\left(\int_{0}^{T} C\left(X_{u}^{*}(t)\right) \mathrm{d} t\right)=\frac{1}{T} \mathrm{E}\left(\int_{0}^{T} C\left(X_{u}^{*}(0)\right) \mathrm{d} t\right)=\mathrm{E}\left(C\left(Z_{u}\right)\right),
$$

and recall that $\mathrm{E}\left(C\left(Z_{u}\right)\right)<\infty$ by (3.7). Then note that, by (3.18),

$$
\begin{aligned}
& \limsup _{T \rightarrow \infty}\left|\frac{1}{T} \mathrm{E}\left(\int_{0}^{T} C\left(X_{u}^{*}(t)\right) \mathrm{d} t\right)-\frac{1}{T} \mathrm{E}\left(\int_{0}^{T} C\left(X_{u}^{x}(t)\right) \mathrm{d} t\right)\right| \\
& \quad \leq \limsup _{T \rightarrow \infty} \frac{1}{T} \mathrm{E}\left(\int_{0}^{\infty}\left|C\left(X_{u}^{x}(t)\right)-C\left(X_{u}^{*}(t)\right)\right| \mathrm{d} t\right) \\
& \quad=0 .
\end{aligned}
$$

Therefore,

$$
\lim _{T \rightarrow \infty} \frac{1}{T} \mathrm{E}\left(\int_{0}^{T} C\left(X_{u}^{x}(t)\right) \mathrm{d} t\right)=\mathrm{E}\left(C\left(Z_{u}\right)\right),
$$

which implies part (ii) of the proposition in view of (3.3). Therefore, the proof of the proposition is complete. 
Remark. The above proposition is in agreement with a result in Theorem 1 of [37] which shows that the limiting distributions of $M(t)=\max _{s \in[0, t]} X_{u}^{x}(s)$ and $M^{*}(t)=\max _{s \in[0, t]} X_{u}^{*}(s)$ coincide, as $t$ tends to $\infty$.

\subsection{Properties of $\mathrm{E}\left(C\left(Z_{u}\right)\right)$}

For $u>0$, let $G(u)=\mathrm{E}\left(C\left(Z_{u}\right)\right)$. We are interested in the behavior of $G(u)$ in view of the identity (3.20).

Lemma 3.2. Let $G(u)=\mathrm{E}\left(C\left(Z_{u}\right)\right)$, where $Z_{u}$ is defined in (3.4). Then the following results hold.

(i) $G(u)$ is a decreasing and continuous function of $u$ on $[0, \infty)$.

(ii) If $C(x)$ is a convex function then so is $G(u)$.

(iii) $\lim _{u \rightarrow 0^{+}} G(u)=+\infty$.

Proof. First we observe that the polynomial bound (2.4) on the growth of $C$ combined with (3.7), which describes the tail behavior of $Z_{u}$, imply that $G(u)=\mathrm{E}\left(C\left(Z_{u}\right)\right)$ is finite for each $u \geq 0$. It is a decreasing function of $u$ because $C$ is nondecreasing while $Z_{u_{1}} \leq Z_{u_{2}}$ if $u_{1}>u_{2}$.

To complete the proof of part (i), it remains to show that $G(u)$ is a continuous function. To prove this, first note that, according to definition (3.4), $Z_{u}$ is a continuous function of the variable $u$ a.s., as shown below. Indeed, if $t_{u}$ is a random time such that $Z_{u}=W_{H}\left(t_{u}\right)-u t_{u}$ and $u \in(0, v)$, then

$$
Z_{u} \geq Z_{v} \geq W_{H}\left(t_{u}\right)-v t_{u}=Z_{u}-t_{u}(v-u) .
$$

Hence, $\lim _{v \rightarrow u^{+}} Z_{v}=Z_{u}$. A similar argument shows that $\lim _{v \rightarrow u^{-}} Z_{v}=Z_{u}$. Therefore, continuity of $C$ implies that $C\left(Z_{u}\right)$ is a continuous function of $u$ with probability 1 . Since $C\left(Z_{u}\right)$ is monotone in $u$, the dominated convergence theorem implies the continuity of $G$.

To prove part (ii), fix constants $r \in[0,1], u_{1}>0$, and $u_{2}>0$, and let $\bar{u}_{r}=r u_{1}+(1-r) u_{2}$. Then

$$
Z_{\bar{u}_{r}}=\sup _{t \geq 0}\left\{W_{H}(t)-\bar{u}_{r} t\right\} \leq r Z_{u_{1}}+(1-r) Z_{u_{2}} .
$$

If $C$ is a nondecreasing convex function, we have

$$
\mathrm{E}\left(C\left(Z_{\bar{u}_{r}}\right)\right) \leq r \mathrm{E}\left(C\left(Z_{u_{1}}\right)\right)+(1-r) \mathrm{E}\left(C\left(Z_{u_{2}}\right)\right) .
$$

Hence, $G$ is convex, and the proof of part (ii) is complete.

Turning to part (iii), we first note that $Z_{0}=\sup _{t \geq 0} W_{H}(t)=+\infty$ with probability 1 . Let $\left(u_{n}\right)_{n \geq 0}$ be any sequence monotonically decreasing to 0 . Then, $Z_{u_{n}}$ is increasing and, hence, there exists a limit (finite or infinite) $\lim _{n \rightarrow \infty} Z_{u_{n}}=L$ and $Z_{u_{n}} \leq L$ for all $n \geq 0$. Thus, $W_{H}(t)-u_{n} t \leq L$ for all $n \geq 0$ and $t \geq 0$. By letting $n$ go to $\infty$ we obtain $\sup _{t \geq 0} W_{H}(t) \leq L$, and, consequently, $L=+\infty$ with probability 1 . Therefore, $\lim _{u \rightarrow 0^{+}} Z(u)=+\infty$ a.s. Since $C$ is a nondecreasing function, the monotone convergence theorem implies that

$$
\lim _{u \rightarrow 0^{+}} \mathrm{E}\left(C\left(Z_{u}\right)\right)=+\infty .
$$

This completes the proof of the lemma. 


\subsection{Existence of an optimal control}

In the following two theorems we provide a representation of the cost functional $I(u, x)$ as well as the existence and uniqueness results for the optimal control $u^{*}>0$.

Theorem 3.1. Let $I(u, x)$ be the cost functional of the long-run average cost problem described in (3.3). Then the following statements hold.

(i) $I(u, x)$ is independent of $x$ and has the representation

$$
I(u):=I(u, x)=h(u)+p u+G(u),
$$

where $G(u)$ is given in Lemma 3.2. Furthermore, $I(u)$ is finite for each $u>0$ and is continuous in $u>0$.

(ii) $\lim _{u \rightarrow 0^{+}} I(u)=+\infty$ and $\lim _{u \rightarrow \infty} I(u)=+\infty$.

(iii) If $h(x)$ and $C(x)$ are convex functions, then $I(u)$ is also convex.

Proof. Part (i) follows from (3.3), Proposition 3.1, and Lemma 3.2.

The first part of claim (ii) follows from the fact that $I(u) \geq G(u)$ along with part (iii) of Lemma 3.2. To verify the second part, note that $I(u) \geq h(u)$ for all $u>0$, and $\lim _{u \rightarrow+\infty} h(u)=$ $+\infty$. Consequently, $\lim _{u \rightarrow+\infty} I(u)=+\infty$.

Part (iii) follows from representation (3.21) combined with the part (ii) of Lemma 3.2. This completes the proof of the theorem.

Theorem 3.2. (i) There is an optimal control $u^{*}>0$ such that, for all $x \geq 0$, we have

$$
I\left(u^{*}\right)=\min _{u>0} I(u, x),
$$

where I is given in (3.21). In particular, $u^{*}$ is independent of $x$.

(ii) In the case $p>0$, if $h$ and $C$ are convex functions, then $u^{*}$ is unique.

(iii) In the case $p=0$, if $h$ is a strictly convex function and $C$ is a convex function, then $u^{*}$ is unique.

Proof. Since $I(u)$ is a continuous function, part (i) follows from parts (i) and (ii) of Theorem 3.1.

If $h$ and $C$ are convex functions, representation (3.21) yields the result that $I$ is a strictly convex function when $p>0$. Therefore, $u^{*}$ is unique in this case.

In the case $p=0$, if $h$ is a strictly convex function and $C$ is a convex function, the result follows from representation (3.21) in a similar way as in the case $p>0$.

\subsection{Generalizations}

In this section we generalize the results in Theorems 3.1 and 3.2 to the more general model introduced in (2.1). Note that, for a fixed control $u>0$ in (2.1), the self-similarity of FBM yields the result that the process $\widehat{W}_{H}$ defined by

$$
\widehat{W}_{H}(t)=\sigma(u) W_{H}\left(\frac{t}{\sigma(u)^{1 / H}}\right), \quad t \in \mathbb{R},
$$

is an FBM. Let

$$
Y_{u}^{x}(t)=X_{u}^{x}\left(\frac{t}{\sigma(u)^{1 / H}}\right)
$$


Then $Y_{u}^{x}$ satisfies

$$
Y_{u}^{x}(t)=x-\frac{u t}{\sigma(u)^{1 / H}}+\widehat{W}_{H}(t)+\widehat{L}_{u}^{x}(t)
$$

where

$$
\widehat{L}_{u}^{x}(t)=L_{u}^{x}\left(\frac{t}{\sigma(u)^{1 / H}}\right) .
$$

Using (2.2) and change of the variable $s=t / \sigma(u)^{1 / H}$, we observe that

$$
\widehat{L}_{u}^{x}(t)=L_{u}^{x}\left(\frac{t}{\sigma(u)^{1 / H}}\right)=-\min \left\{0, \min _{s \in[0, t]}\left(x-\frac{s u}{\sigma(u)^{1 / H}}+\widehat{W}_{H}(s)\right)\right\} .
$$

Equations (3.22) and (3.23) are analogous to (2.1) and (2.2).

We next consider the change in the cost structure due to the change of the variable $s=$ $t / \sigma(u)^{1 / H}$. We note that

$$
\frac{1}{T} \int_{0}^{T} C\left(X_{u}^{x}(t)\right) \mathrm{d} t=\frac{1}{M(T)} \int_{0}^{M(T)} C\left(Y_{u}^{x}(t)\right) \mathrm{d} t
$$

where $M(T)=\sigma(u)^{1 / H} T$. Therefore, using the results in Theorem 3.1, we obtain

$$
\lim _{T \rightarrow \infty} \frac{1}{T} \mathrm{E}\left(\int_{0}^{T} C\left(X_{u}^{x}(t)\right) \mathrm{d} t\right)=G\left(\frac{u}{\sigma(u)^{1 / H}}\right) .
$$

We have the following result.

Theorem 3.3. Consider the controlled state process $X_{u}^{x}$ defined by (2.1) and (2.2) with the cost functional $I(x, u)$ given in $(2.5)$. Define $f:[0, \infty) \rightarrow[0, \infty)$ by

$$
f(u)=\frac{u}{\sigma(u)^{1 / H}} .
$$

Then

$$
I(u, x)=h(u)+p u+G(f(u)),
$$

where the function $G$ is given in Lemma 3.2. Furthermore, $I(u, x)$ is independent of $x$ (we will henceforth denote the cost function by $I(u))$.

Proof. The same argument as that used in the proof of Lemma 3.1 yields

$$
\lim _{T \rightarrow \infty} \frac{1}{T} \mathrm{E}\left(L_{u}^{x}(T)\right)=u .
$$

Combining this result with (3.24) we obtain representation (3.26).

Our next result is analogous to Theorems 3.1 and 3.2.

Theorem 3.4. Assume that the function $f$ in (3.25) is continuous and that $\lim _{u \rightarrow 0^{+}} f(u)=0$. Then, with $I(u)=I(u, x)$ as in (3.26), the following statements hold.

(i) $\lim _{u \rightarrow 0^{+}} I(u)=\lim _{u \rightarrow+\infty} I(u)=+\infty$, and $I(u)$ is a finite continuous function on $[0, \infty)$. Furthermore, there is a constant $u^{*}>0$ such that $I\left(u^{*}\right)=\min _{u>0} I(u)$.

(ii) If $f$ is a concave increasing function then statements similar to parts (i) and (ii) of Theorem 3.2 (regarding the uniqueness $u^{*}$ ) hold. 
The proof of this theorem is a straightforward modification of the proofs of Theorems 3.1 and 3.2, and is therefore omitted.

Remark. We can further generalize our model to cover the following situation. For given positive continuous functions $b(u)$ and $\sigma(u)$, let

$$
X_{u}^{x}(t)=x+\sigma(u) W_{h}(t)-b(u) t+L_{u}^{x}(t)
$$

where, for $u>0$,

$$
L_{u}^{x}(t)=-\min \left\{0, \min _{s \in[0, t]}\left(x-b(u) s+\sigma(u) W_{H}(s)\right)\right\} .
$$

The optimization problem here is to minimize the cost functional $I(u, x)$ defined in (2.5).

Following the time change method described in Section 3.5, we can obtain an analogue of Theorem 3.4 regarding the derivation of the optimal control. In this situation, the function $f$ defined in (3.25) needs to be replaced by $f(u)=b(u)(\sigma(u))^{-1 / H}$ with the assumptions that $f$ is continuous and $\lim _{u \rightarrow 0^{+}} f(u)=0$. We omit the details of the proof.

\section{A constrained minimization problem}

In this section we address a constrained minimization problem that can be solved by using our results in Section 3. Let $\left\{W_{H}(t): t \geq 0\right\}$ be an FBM defined on a complete probability space $(\Omega, \mathcal{F}, \mathrm{P})$. Our model here is of the form

$$
Y_{u}^{x}(t)=x-u t+\sigma(u) W_{H}(t)+K_{u}^{x}(t)
$$

where $\sigma$ is a nonnegative continuous function, $K_{u}^{x}(\cdot)$ is a nonnegative, nondecreasing rightcontinuous with left limits (RCLL) process adapted to the natural filtration $\left(\mathcal{F}_{t}\right)_{t \geq 0}$, where $\mathcal{F}_{t}$ is the $\sigma$-algebra generated by $\left\{W_{H}(s): 0 \leq s \leq t\right\}$, augmented with all the null sets in $\mathcal{F}$. Furthermore, $K_{u}^{x}(0)=0$ and the process $K_{u}^{x}$ is chosen by the controller in such a way that the state process $Y_{u}^{x}$ is constrained to nonnegative reals. In this situation, the controller is equipped with two controls: the choice of $u>0$ and the choice of the $K_{u}^{x}$ process subject to the nonnegativity of the $Y_{u}^{x}$ process.

Throughout this section, we keep the initial state $x \geq 0$ fixed. We will deduce using the results of the previous section that the value of this minimization problem as well as the optimal control are not affected by the initial data.

Let $m>0$ be any fixed positive constant. The constrained minimization problem we would like to address here is the following.

$$
\begin{array}{ll}
\text { Minimize } & \limsup _{T \rightarrow \infty} \frac{1}{T} \mathrm{E}\left(\int_{0}^{T}\left[h(u)+C\left(Y_{u}^{x}(t)\right)\right] \mathrm{d} t\right) \\
\text { Subject to } & \limsup _{T \rightarrow \infty} \frac{\mathrm{E}\left(K_{u}^{x}(T)\right)}{T} \leq m,
\end{array}
$$

over all feasible controls $u>0$ and feasible processes $K_{u}^{x}(\cdot)$ which ensure that $Y_{u}^{x}(\cdot)$ is a nonnegative process. A controlled optimization problem of this nature for diffusion processes was considered in [1], and for a more complete treatment in the case of diffusion processes, we refer the reader to [32]. 
Fix any integer $m>0$, and define a class of state processes $U_{m}$ as follows:

$$
u_{m}=\left\{\left(Y_{u}^{x}, K_{u}^{x}\right): Y_{u}^{x}(t) \geq 0 \text { for } t \geq 0,(4.1) \text { is satisfied, } \limsup _{T \rightarrow \infty} \frac{\mathrm{E}\left(K_{u}^{x}(T)\right)}{T} \leq m\right\} .
$$

From our results in Section 3, it follows that, for any $u \leq m$, the pair $\left(X_{u}^{x}, L_{u}^{x}\right)$ in (2.1) and (2.2) belongs to $U_{m}$, and, hence, $U_{m}$ is nonempty. Therefore, the constrained minimization problem is to find

$$
\inf _{\left(Y_{u}^{x}, K_{u}^{x}\right) \in U_{m}} \limsup _{T \rightarrow \infty} \frac{1}{T} \mathrm{E}\left(\int_{0}^{T}\left[h(u)+C\left(Y_{u}^{x}(t)\right)\right] \mathrm{d} t\right) .
$$

In this section we make the following additional assumptions.

(i) For functions $h$ and $C$, we assume that

$h$ is strictly convex and satisfies (2.3), $C$ is convex and satisfies (2.4).

(ii) Let $f(u)=u / \sigma(u)^{1 / H}$. We assume that

$$
f(u)>0, \lim _{u \rightarrow 0^{+}} f(u)=0 \text {, and } f \text { is a convex increasing function. }
$$

The following lemma enables us to reduce the set $U_{m}$ to the collection of processes $\left(X_{u}^{x}, L_{u}^{x}\right)$ described in the previous section, with $u \leq m$.

Lemma 4.1. Let $u>0$, and let $\left(Y_{u}^{x}, K_{u}^{x}\right)$ be a pair of processes satisfying (4.1). Consider $\left(X_{u}^{x}, L_{u}^{x}\right)$, which satisfies (2.1) and (2.2), and is defined on the same probability space as $\left(Y_{u}^{x}, K_{u}^{x}\right)$. Then

(i) $L_{u}^{x}(t) \leq K_{u}^{x}(t)$ and $X_{u}^{x}(t) \leq Y_{u}^{x}(t)$ for $t \geq 0$.

(ii) $u=\lim _{T \rightarrow \infty} \frac{1}{T} \mathrm{E}\left(L_{u}^{x}(T)\right) \leq \limsup _{T \rightarrow \infty} \frac{1}{T} \mathrm{E}\left(K_{u}^{x}(T)\right)$.

(iii) $G(f(u))=\lim _{T \rightarrow \infty} \frac{1}{T} \mathrm{E}\left(\int_{0}^{T} C\left(X_{u}^{x}(t)\right) \mathrm{d} t\right) \leq \limsup _{T \rightarrow \infty} \frac{1}{T} \mathrm{E}\left(\int_{0}^{T} C\left(Y_{u}^{x}(t)\right) \mathrm{d} t\right)$.

Proof. Since $Y_{u}^{x} \geq 0, K_{u}^{x}(0)=0$, and $K_{u}^{x}$ is a nonincreasing process, the minimality property of the reflection map stated in (2.16) implies that $L_{u}^{x}(t) \leq K_{u}^{x}(t)$ and $X_{u}^{x}(t) \leq Y_{u}^{x}(t)$ for $t \geq 0$.

Next, observe that part (ii) of the lemma follows from the result in part (i) while the identity $u=\lim _{T \rightarrow \infty}(1 / T) \mathrm{E}\left(L_{u}^{x}(T)\right)$ is implied by Lemma 3.1 (see also the remark immediately after the proof of Lemma 3.1).

Finally, part (iii) of the lemma follows from (3.24), part (i), and from the fact that $C$ is nondecreasing. The proof of the lemma is complete.

Let

$$
\mathcal{V}_{m}=\left\{\left(X_{u}^{x}, L_{u}^{x}\right):(2.1) \text { and (2.2) are satisfied, and, in addition, } u \leq m\right\} .
$$

From the above lemma, it is clear that

$$
\begin{aligned}
& \inf _{\left(K_{u}^{x}, Y_{u}^{x}\right) \in \mathcal{U}_{m}} \lim _{T \rightarrow \infty} \frac{1}{T} \mathrm{E}\left(\int_{0}^{T}\left[h(u)+C\left(Y_{u}^{x}(t)\right)\right] \mathrm{d} t\right) \\
& =\inf _{\left(X_{u}^{x}, L_{u}^{x}\right) \in \mathcal{V}_{m}} \limsup _{T \rightarrow \infty} \frac{1}{T} \mathrm{E}\left(\int_{0}^{T}\left[h(u)+C\left(X_{u}^{x}(t)\right)\right] \mathrm{d} t\right) .
\end{aligned}
$$


Therefore, our minimization problem is reduced. Next, we can use the results in Section 3.5 and write, for any $u>0$,

$$
\lim _{T \rightarrow \infty} \frac{1}{T} \mathrm{E}\left(\int_{0}^{T}\left[h(u)+C\left(X_{u}^{x}(t)\right)\right] \mathrm{d} t\right)=h(u)+G(f(u)) .
$$

Here $G$ is given by Lemma 3.2, and $f$ is described in (3.25) and (4.5). Consequently,

$$
\inf _{\left(X_{u}^{x}, L_{u}^{x}\right) \in \mathcal{V}_{m}} \lim _{T \rightarrow \infty} \frac{1}{T} \mathrm{E}\left(\int_{0}^{T}\left[h(u)+C\left(X_{u}^{x}(t)\right)\right] \mathrm{d} t\right)=\inf \{h(u)+G(f(u)): 0<u \leq m\} .
$$

We next consider the optimal control described in Theorem 3.4 corresponding to the case $p=0$. In virtue of assumptions (4.4) and (4.5), the optimal control is unique and we will label it by $u_{0}^{*}>0$. We have the following result.

Theorem 4.1. Let

$$
u^{*}(m)= \begin{cases}m & \text { if } m<u_{0}^{*} \\ u_{0}^{*} & \text { if } m \geq u_{0}^{*}\end{cases}
$$

where $u_{0}^{*}$ is the unique optimal control in Theorem 3.4 corresponding to $p=0$.

Then the pair $\left(X_{x}^{u^{*}(m)}, L_{x}^{u^{*}(m)}\right)$ is an optimal process for the constrained minimization problem (4.2) and (4.3). Furthermore, the optimal control $u^{*}(m)$ is a continuous increasing function of the parameter $m$.

Proof. Let $\Lambda(u)=h(u)+G(f(u))$, where $f$ and $G$ are as in (4.7). Then, by assumptions (4.4) and (4.5), and Theorem 3.4, $\Lambda$ is a strictly convex function which is finite everywhere on $(0, \infty)$. Furthermore, $\lim _{u \rightarrow 0^{+}} \Lambda(u)=+\infty$ and $\lim _{u \rightarrow+\infty} \Lambda(u)=+\infty$, and, hence, $\Lambda$ has a unique minimum at $u_{0}^{*}$. Therefore, $\Lambda$ is strictly increasing on $\left(u_{0}^{*}, \infty\right)$. Clearly, with $u^{*}(m)$ defined in (4.9),

$$
\Lambda\left(u^{*}(m)\right)=\inf _{u \leq m} \Lambda(u),
$$

and $u^{*}(m)$ is the unique number which has this property. By (4.6) and (4.8), we have

$$
\Lambda\left(u^{*}(m)\right)=\inf _{\left(X_{u}^{x}, L_{u}^{x}\right) \in \mathcal{V}_{m}} \limsup _{T \rightarrow \infty} \frac{1}{T} \mathrm{E}\left(\int_{0}^{T}\left[h(u)+C\left(Y_{u}^{x}(t)\right)\right] \mathrm{d} t\right) .
$$

Consider the pair of processes $\left(X_{u^{*}(m)}^{x}, L_{u^{*}(m)}^{x}\right)$ defined in (2.1) and (2.2). Then, by virtue of Lemma 3.1, we have

$$
\lim _{T \rightarrow \infty} \frac{1}{T} \mathrm{E}\left(L_{u^{*}(m)}^{x}(T)\right)=u^{*}(m) \leq m
$$

and, by Theorem 3.6,

$$
\limsup _{T \rightarrow \infty} \frac{1}{T} \mathrm{E}\left(\int_{0}^{T}\left[h(u)+C\left(X_{u^{*}(m)}^{x}(t)\right)\right] \mathrm{d} t\right)=\Lambda\left(u^{*}(m)\right) .
$$

Hence, $\left(X_{u^{*}(m)}^{x}, L_{u^{*}(m)}^{x}\right)$ describes an optimal strategy. This completes the proof of the theorem.

Remark. Note that the above optimal control $u^{*}(m)$ is independent of the initial point $x$. 


\section{Infinite-horizon discounted cost minimization problem}

In this section we define an optimal control $u^{*}$ for the infinite-horizon discounted cost functional given in (2.7). Throughout this section, we assume that $\sigma(u) \equiv 1$, the state process $X_{u}^{x}$ satisfies (3.1), and that the functionals $h$ and $C$ are convex in addition to the assumptions stated in (2.3) and (2.4). In contrast with Section 3, our methods here do not readily extend to the case where the function $\sigma(u)$ is nonconstant.

The discounted cost functional $J_{\alpha}(x, u)$ is given by

$$
\begin{aligned}
J_{\alpha}(x, u) & =\mathrm{E}\left(\int_{0}^{\infty} \mathrm{e}^{-\alpha t}\left[h(u)+C\left(X_{u}^{x}(t)\right)\right] \mathrm{d} t+\int_{0}^{\infty} \mathrm{e}^{-\alpha t} p \mathrm{~d} L_{u}^{x}(t)\right) \\
& =\frac{h(u)}{\alpha}+\mathrm{E}\left(\int_{0}^{\infty} \mathrm{e}^{-\alpha t}\left[C\left(X_{u}^{x}(t)\right)+\alpha p L_{u}^{x}(t)\right] \mathrm{d} t\right) .
\end{aligned}
$$

Here $\alpha>0$ is a constant discount factor and $p>0$ is also a constant. To derive the last equality above, we used Fubini's theorem to obtain $\int_{0}^{\infty} \mathrm{e}^{-\alpha t} \mathrm{~d} L_{u}^{x}(t)=\alpha \int_{0}^{\infty} \mathrm{e}^{-\alpha t} L_{u}^{x}(t) \mathrm{d} t$.

Let

$$
J_{\alpha, 1}(x, u)=\mathrm{E}\left(\int_{0}^{\infty} \mathrm{e}^{-\alpha t} C\left(X_{u}^{x}(t)\right) \mathrm{d} t\right)
$$

and

$$
J_{\alpha, 2}(x, u)=\mathrm{E}\left(\int_{0}^{\infty} \mathrm{e}^{-\alpha t} L_{u}^{x}(t) \mathrm{d} t\right) .
$$

Next we use the convexity of the reflection mapping described in (2.15) to establish the convexity of the cost functional with respect to $u$.

Lemma 5.1. Let $x \geq 0$ be fixed, and let $C$ be a convex function satisfying assumption (2.4). Then, $J_{\alpha, 1}(x, u)$ and $J_{\alpha, 2}(x, u)$ introduced above are finite for each $u \geq 0$ and are convex in the $u$ variable.

Proof. By (3.2) we have the bound $\mathrm{E}\left(L_{u}^{x}(t)\right) \leq u t+K_{0}\left(1+t^{H}\right)$, where $K_{0}>0$ is a constant independent of $t$. This implies, using Fubini's theorem, that $J_{\alpha, 2}(x, u)$ is finite.

Next, using (3.18), we obtain

$$
\left|J_{\alpha, 1}(x, u)-\mathrm{E}\left(\int_{0}^{\infty} \mathrm{e}^{-\alpha t} C\left(X_{u}^{*}(t)\right) \mathrm{d} t\right)\right| \leq \mathrm{E}\left(\int_{0}^{\infty}\left|C\left(X_{u}^{x}(t)\right)-C\left(X_{u}^{*}(t)\right)\right| \mathrm{d} t\right)<\infty .
$$

But, using the stationarity of $X_{u}^{*}$, we have $\mathrm{E}\left(\int_{0}^{\infty} \mathrm{e}^{-\alpha t} C\left(X_{u}^{*}(t)\right) \mathrm{d} t\right)=\alpha^{-1} \mathrm{E}\left(C\left(Z_{u}\right)\right)$, where $Z_{u}$ is given in (3.4). Note that $\mathrm{E}\left(C\left(Z_{u}\right)\right)$ is finite because $C$ has polynomial growth and by virtue of (3.7). Consequently, $J_{\alpha, 1}(x, u)$ is also finite.

To establish convexity of $J_{\alpha, 1}(x, u)$, first recall that $X_{u}^{x}=\Gamma\left(x+W_{H}-u e\right)$, where $e(t) \equiv t$ for $t \geq 0$, and $\Gamma$ is the reflection mapping described in Section 2.3. Now let $u_{1} \geq 0, u_{2} \geq 0$, and $r \in(0,1)$. Then, for $t \geq 0$,

$$
\begin{aligned}
x+ & W_{H}(t)-\left(r u_{1}+(1-r) u_{2}\right) e(t) \\
& =r\left(x+W_{H}(t)-u_{1} e(t)\right)+(1-r)\left(x+W_{H}(t)-u_{2} e(t)\right) .
\end{aligned}
$$

Since the reflection map $\Gamma$ satisfies the convexity property (2.15), we have

$$
X_{\bar{u}_{r}}^{x}(t) \leq r X_{u_{1}}^{x}(t)+(1-r) X_{u_{2}}^{x}(t),
$$

where $\bar{u}_{r}=r u_{1}+(1-r) u_{2}$. Next, since $C$ is a convex nondecreasing function, (5.3) implies 
that $C\left(X_{\bar{u}_{r}}^{x}(t)\right) \leq r C\left(X_{u_{1}}^{x}(t)\right)+(1-r) C\left(X_{u_{2}}^{x}(t)\right)$ for $t \geq 0$. From this, it follows that

$$
J_{\alpha, 1}\left(x, \bar{u}_{r}\right) \leq r J_{\alpha, 1}\left(x, u_{1}\right)+(1-r) J_{\alpha, 1}\left(x, u_{2}\right) .
$$

Hence, $J_{\alpha, 1}(x, u)$ is convex in the $u$ variable.

Next, by (2.2) and (2.11), we have

$$
\Gamma\left(x+W_{H}-u e\right)(t)-\left(x+W_{H}-u e\right)(t)=L_{u}^{x}(t), \quad t \geq 0 .
$$

Then, since $\Gamma$ is convex in the $u$ variable by (2.15), the process $L_{u}^{x}$ is also convex in the $u$ variable. Consequently, with $\bar{u}_{r}=r u_{1}+(1-r) u_{2}$, we obtain

$$
L_{\bar{u}_{r}}^{x}(t) \leq r L_{u_{1}}^{x}(t)+(1-r) L_{u_{2}}^{x}(t), \quad t \geq 0 .
$$

Finally, it is evident that $J_{\alpha, 2}(x, u)$ is convex in the $u$ variable from definition (5.2).

Corollary 5.1. Under the conditions of Lemma 5.1, the discounted cost functional $J_{\alpha}(x, u)$ is finite for each $u \geq 0$ and is convex in the $u$ variable.

Proof. Note that

$$
J_{\alpha}(x, u)=\frac{h(u)}{\alpha}+J_{\alpha, 1}(x, u)+\alpha p J_{\alpha, 2}(x, u) .
$$

By our assumptions, $h$ is a convex function, and $p \geq 0$ and $\alpha>0$ are constants. Therefore, the claim follows from Lemma 5.1.

The above lemma and corollary lead to the following result.

Theorem 5.1. Consider the $X_{u}^{x}$ process satisfying (3.1) and the associated discounted cost functional $J_{\alpha}(x, u)$ described in (5.1). Then, for each initial point $x \geq 0$, there is an optimal control $u^{*} \geq 0$ such that

$$
J_{\alpha}\left(x, u^{*}\right)=\inf _{u \geq 0} J_{\alpha}(x, u) \equiv V_{\alpha}(x),
$$

where $V_{\alpha}(x)$ is the value function of the discounted cost problem defined in (2.8).

Proof. Fix any $x \geq 0$ and $\alpha>0$. By Corollary 5.1, $J_{\alpha}(x, u)$ is finite for each $u \geq 0$ and is convex in the $u$ variable. By (5.4), we have $J_{\alpha}(x, u) \geq h(u) / \alpha$ and hence, since $\lim _{u \rightarrow \infty} h(u)=+\infty$, we obtain $\lim _{u \rightarrow \infty} J_{\alpha}(x, u)=+\infty$. Since $J_{\alpha}(x, u)$ is convex in the $u$ variable, we can conclude that there is a $u^{*} \geq 0$ (which may depend on $x$ ) such that $J_{\alpha}\left(x, u^{*}\right)=\inf _{u \geq 0} J_{\alpha}(x, u)$. This completes the proof of the theorem.

Remark. Note that in contrast with the long-run average cost minimization problem, we cannot rule out the possibility that $u^{*}=0$ here.

Corollary 5.2. For the special case $p=0$, assume further that $h(x)$ is constant on an interval $[0, \delta]$ for some $\delta>0$. Then, for every initial point $x \geq 0$, the optimal control $u^{*}$ is strictly positive.

Proof. It follows from (5.4) that $J_{\alpha}(x, u)=J_{\alpha, 1}(x, u)$. The function $C$ is increasing and $X_{u_{1}}^{x}(t)<X_{u_{2}}^{x}(t)$ for all $t \geq 0$ and $u_{1}>u_{2}$. Therefore $J_{\alpha, 1}\left(x, u_{1}\right) \leq J_{\alpha, 1}\left(x, u_{2}\right)$ for $u_{1}>u_{2}$. Consequently, $J_{\alpha}(x, 0) \geq J_{\alpha}(x, u)$ for all $u>0$. Hence, we can find an optimal control $u^{*}>0$, and the proof of the corollary is complete. 


\section{Finite-horizon problem and Abelian limits}

In this section we establish Abelian limit relationships among the value functions of three stochastic control problems introduced in Section 2.2. The main result is stated in Theorem 6.1, below. Throughout this section, for simplicity, we assume that $\sigma(u) \equiv 1$ in the model described in (2.1).

We begin with the existence of an optimal control for the finite-horizon control problem introduced in Section 2.2.

Proposition 6.1. Let $x \geq 0, T>0$, and $I(u, x, T)$ be defined by (2.10). Then

(i) $I(u, x, T)$ is finite for each $u>0$ and is continuous in $u>0$,

(ii) $\lim _{u \rightarrow \infty} I(u, x, T)=+\infty$,

(iii) if $h$ and $C$ are convex functions, $I(u, x, T)$ is a convex function of the variable $u$.

Corollary 6.1. For any fixed $x \geq 0$ and $T>0$, the following statements hold.

(i) There is an optimal control $u^{*}(x, T) \geq 0$ such that $I\left(u^{*}(x, T)\right)=\min _{u>0} I(u, x, T)$.

(ii) In the case $p>0$, if $h$ and $C$ are convex functions, then $u^{*}(x, T)$ is unique.

(iii) In the case $p=0$, if $h$ is a strictly convex function and $C$ is a convex function, then $u^{*}(x, T)$ is unique.

The proofs of the above proposition and corollary are straightforward adaptations of the corresponding proofs given in Section 3, and are therefore omitted.

The following theorem is the main result of this section.

Theorem 6.1. Let $X_{u}^{x}$ satisfy (3.1) and let $V_{0}, V_{\alpha}(x)$, and $V(x, T)$ be the value functions defined in (2.6), (2.8), and (2.9), respectively. Then the following Abelian limit relationships hold:

$$
\lim _{\alpha \rightarrow 0^{+}} \alpha V_{\alpha}(x)=\lim _{T \rightarrow \infty} \frac{V(x, T)}{T}=V_{0} .
$$

We prove this result in Propositions 6.2 and 6.3, below. The following technical lemma gathers necessary tools to establish $\lim _{\alpha \rightarrow 0^{+}} \alpha V_{\alpha}(x)=V_{0}$.

Lemma 6.1. Let $u>0$ be given, and let $X_{u}^{x}$ satisfy (3.1). Consider the cost functional $J_{\alpha}(x, u)$ as defined in (2.7). Then

$$
\lim _{\alpha \rightarrow 0^{+}} \alpha J_{\alpha}(x, u)=I(u),
$$

where I $(u)$ is described in (3.21).

Proof. First consider $\lim _{\alpha \rightarrow 0^{+}} \alpha \mathrm{E}\left(\int_{0}^{\infty} \mathrm{e}^{-\alpha t} \mathrm{~d} L_{u}^{x}(t)\right)$, where $L_{u}^{x}$ is as in (2.2). Similarly to (5.1), we have

$$
\alpha \mathrm{E}\left(\int_{0}^{\infty} \mathrm{e}^{-\alpha t} \mathrm{~d} L_{u}^{x}(t)\right)=\alpha^{2} \mathrm{E}\left(\int_{0}^{\infty} \mathrm{e}^{-\alpha t} L_{u}^{x}(t) \mathrm{d} t\right)=\alpha^{2} \int_{0}^{\infty} \mathrm{e}^{-\alpha t} \mathrm{E}\left(L_{u}^{x}(t)\right) \mathrm{d} t,
$$

where we used Fubini's theorem to obtain the last identity. By (3.1),

$$
\mathrm{E}\left(L_{u}^{x}(t)\right)=u t+\mathrm{E}\left(X_{u}^{x}(t)\right)-x .
$$


Therefore,

$$
\begin{aligned}
\alpha^{2} \int_{0}^{\infty} \mathrm{e}^{-\alpha t} \mathrm{E}\left(L_{u}^{x}(t)\right) \mathrm{d} t & =\alpha^{2} u \int_{0}^{\infty} \mathrm{e}^{-\alpha t} t \mathrm{~d} t+\alpha^{2} \int_{0}^{\infty} \mathrm{e}^{-\alpha t} \mathrm{E}\left(X_{u}^{x}(t)\right) \mathrm{d} t-\alpha x \\
& =u+\alpha^{2} \int_{0}^{\infty} \mathrm{e}^{-\alpha t} \mathrm{E}\left(X_{u}^{x}(t)\right) \mathrm{d} t-\alpha x
\end{aligned}
$$

Furthermore, by (3.2), $0 \leq \mathrm{E}\left(X_{u}^{x}(t)\right) \leq K_{0}\left(1+t^{H}\right)$, where the constant $K_{0}>0$ is independent of $t$ and $u$. Thus,

$$
\begin{aligned}
0 & \leq \alpha^{2} \int_{0}^{\infty} \mathrm{e}^{-\alpha t} \mathrm{E}\left(X_{u}^{x}(t)\right) \mathrm{d} t \\
& \leq K_{0} \alpha^{2} \int_{0}^{\infty} \mathrm{e}^{-\alpha t}\left(1+t^{H}\right) \mathrm{d} t \\
& \leq K_{0}\left(\alpha+\operatorname{gamma}(H) \alpha^{1-H}\right),
\end{aligned}
$$

where $\operatorname{gamma}(H):=\int_{0}^{\infty} \mathrm{e}^{-t} t^{H} \mathrm{~d} t=\alpha^{1+H} \int_{0}^{\infty} \mathrm{e}^{-\alpha t} t^{H} \mathrm{~d} t$ is the gamma function evaluated at $H$.

Since $H \in(0,1)$, it follows from (6.3) that $\lim _{\alpha \rightarrow 0^{+}} \alpha^{2} \int_{0}^{\infty} \mathrm{e}^{-\alpha t} \mathrm{E}\left(X_{u}^{x}(t)\right) \mathrm{d} t=0$. Hence, using (6.1) and (6.2), we obtain

$$
\lim _{\alpha \rightarrow 0^{+}} \alpha \mathrm{E}\left(\int_{0}^{\infty} \mathrm{e}^{-\alpha t} \mathrm{~d} L_{u}^{x}(t)\right)=u .
$$

We next consider $\lim _{\alpha \rightarrow 0^{+}} \alpha \mathrm{E}\left(\int_{0}^{\infty} \mathrm{e}^{-\alpha t} C\left(X_{u}^{x}(t)\right) \mathrm{d} t\right)$. It follows from (3.18) that

$$
\begin{aligned}
& \left|\mathrm{E}\left(\int_{0}^{\infty} \mathrm{e}^{-\alpha t} C\left(X_{u}^{x}(t)\right) \mathrm{d} t\right)-\mathrm{E}\left(\int_{0}^{\infty} \mathrm{e}^{-\alpha t} C\left(X_{u}^{*}(t)\right) \mathrm{d} t\right)\right| \\
& \quad \leq \mathrm{E}\left(\int_{0}^{\infty}\left|C\left(X_{u}^{x}(t)\right)-C\left(X_{u}^{*}(t)\right)\right| \mathrm{d} t\right) \\
& \quad<\infty
\end{aligned}
$$

where $X_{u}^{*}$ is the stationary process described in (3.5) and (3.6). Therefore,

$$
\begin{aligned}
\lim _{\alpha \rightarrow 0^{+}} \alpha \mathrm{E}\left(\int_{0}^{\infty} \mathrm{e}^{-\alpha t} C\left(X_{u}^{x}(t)\right) \mathrm{d} t\right) & =\lim _{\alpha \rightarrow 0^{+}} \alpha \mathrm{E}\left(\int_{0}^{\infty} \mathrm{e}^{-\alpha t} C\left(X_{u}^{*}(t)\right) \mathrm{d} t\right) \\
& =\lim _{\alpha \rightarrow 0^{+}} \alpha \int_{0}^{\infty} \mathrm{e}^{-\alpha t} \mathrm{E}\left(C\left(Z_{u}\right)\right) \mathrm{d} t \\
& =\mathrm{E}\left(C\left(Z_{u}\right)\right) \\
& =G(u),
\end{aligned}
$$

where $G(u)$ is defined in Section 3.3. It follows from (2.7), (6.4), and (6.5) that

$$
\lim _{\alpha \rightarrow 0^{+}} \alpha J_{\alpha}(x, u)=h(u)+p u+G(u)=I(u),
$$

where $I(u)$ is given in (3.21). This completes the proof of the lemma.

The next proposition contains the proof of the first part of Theorem 6.1. 
Proposition 6.2. Let $X_{u}^{x}$ satisfy (3.1), and let $V_{\alpha}(x)$ be the corresponding value function defined in (2.8). Then

$$
\lim _{\alpha \rightarrow 0^{+}} \alpha V_{\alpha}(x)=V_{0}<\infty,
$$

where $V_{0}$ is the value of the long-run average cost minimization problem given in (2.6).

Proof. Fix the initial point $x \geq 0$. For any $u>0$, we have $\alpha V_{\alpha}(x) \leq \alpha J_{\alpha}(x, u)$. Hence, by Lemma 6.1, $\lim \sup _{\alpha \rightarrow 0^{+}} \alpha V_{\alpha}(x) \leq \lim _{\alpha \rightarrow 0^{+}} \alpha J_{\alpha}(x, u)=I(u)$. Therefore, minimizing the right-hand side over $u>0$, we obtain

$$
\limsup _{\alpha \rightarrow 0^{+}} \alpha V_{\alpha}(x) \leq \inf _{u>0} I(u)=V_{0} .
$$

It remains to show the validity of the reverse inequality, namely that

$$
\liminf _{\alpha \rightarrow 0^{+}} \alpha V_{\alpha}(x) \geq \inf _{u>0} I(u)=V_{0} .
$$

To this end, consider a decreasing to zero sequence $\alpha_{n}>0, n \in \mathbb{N}$, such that

$$
\liminf _{\alpha \rightarrow 0^{+}} \alpha V_{\alpha}(x)=\lim _{n \rightarrow \infty} \alpha_{n} V_{\alpha_{n}}(x)
$$

Fix any $\varepsilon_{0}>0$, and let $u_{n}>0, n \in \mathbb{N}$, be a sequence such that $V_{\alpha_{n}}(x)+\varepsilon_{0}>J_{\alpha_{n}}\left(x, u_{n}\right)$. Then

$$
\alpha_{n} V_{\alpha_{n}}(x)+\alpha_{n} \varepsilon_{0}>\alpha_{n} J_{\alpha_{n}}\left(x, u_{n}\right) \geq h\left(u_{n}\right) .
$$

Letting $n \rightarrow \infty$ we obtain

$$
\limsup _{n \rightarrow \infty} h\left(u_{n}\right) \leq \limsup _{n \rightarrow \infty} \alpha_{n} V_{\alpha_{n}}(x) \leq V_{0} .
$$

Since $\lim _{x \rightarrow \infty} h(x)=+\infty$, this implies that $u_{n}$ is a bounded sequence. That is, there is an $M>0$ such that $u_{n} \in(0, M)$ for all $n \in \mathbb{N}$. Therefore, without loss of generality, we can assume that $u_{n}$ converges as $n \rightarrow \infty$ to some $u_{\infty} \in[0, M]$ (otherwise, we can consider a convergent subsequence of $u_{n}$ ).

Let $\delta \in\left(u_{\infty}, \infty\right)$. Then,

$$
\alpha_{n} J_{\alpha_{n}}\left(x, u_{n}\right) \geq h\left(u_{n}\right)+\alpha_{n}^{2} p \int_{0}^{\infty} \mathrm{e}^{-\alpha_{n} t} \mathrm{E}\left(L_{u_{n}}^{x}(t)\right) \mathrm{d} t+\alpha_{n} \int_{0}^{\infty} \mathrm{e}^{-\alpha_{n} t} \mathrm{E}\left[C\left(X_{\delta}^{x}(t)\right)\right] \mathrm{d} t .
$$

Since $\mathrm{E}\left(L_{u_{n}}^{x}(t)\right)=\mathrm{E}\left(X_{u_{n}}^{x}(t)\right)+u_{n} t-x \geq u_{n} t-x$, we obtain

$$
\alpha_{n}^{2} \int_{0}^{\infty} \mathrm{e}^{-\alpha_{n} t} \mathrm{E}\left(L_{u_{n}}^{x}(t)\right) \mathrm{d} t \geq u_{n}-\alpha_{n} x
$$

and, hence,

$$
\alpha_{n} J_{\alpha_{n}}\left(x, u_{n}\right) \geq h\left(u_{n}\right)+p u_{n}-p \alpha_{n} x+\alpha_{n} \int_{0}^{\infty} \mathrm{e}^{-\alpha_{n} t} \mathrm{E}\left[C\left(X_{\delta}^{x}(t)\right)\right] \mathrm{d} t .
$$

Therefore, letting $n$ go to $\infty$ and using (6.5), we obtain

$$
\liminf _{n \rightarrow \infty} \alpha_{n} J_{\alpha_{n}}\left(x, u_{n}\right) \geq h\left(u_{\infty}\right)+p u_{\infty}+\mathrm{E}\left[C\left(Z_{\delta}\right)\right] .
$$


In particular, we can conclude that $u_{\infty}>0$, because otherwise, letting $\delta$ tend to 0 and using Lemma 3.2, we would obtain $\lim _{n \rightarrow \infty} \inf _{n \rightarrow \infty} J_{\alpha_{n}}\left(x, u_{n}\right)=+\infty$, which contradicts the fact that $\lim \sup _{n \rightarrow \infty} \alpha_{n} J_{\alpha_{n}}\left(x, u_{n}\right) \leq V_{0}<\infty$ according to (6.8) and (6.9). Therefore, $u_{\infty}>0$.

Letting $\delta$ in (6.10) tend to $u_{\infty}$ and again using the continuity of $G(u)=\mathrm{E}\left[C\left(Z_{u}\right)\right]$ proved in Lemma 3.2, we obtain

$$
\liminf _{n \rightarrow \infty} \alpha_{n} J_{\alpha_{n}}\left(x, u_{n}\right) \geq h\left(u_{\infty}\right)+p u_{\infty}+\mathrm{E}\left[C\left(Z_{u_{\infty}}\right)\right]=I\left(u_{\infty}\right) \geq V_{0}
$$

Inequalities (6.8), (6.9), and (6.11) combined together yield

$$
\lim _{n \rightarrow \infty} \alpha_{n} J_{\alpha_{n}}\left(x, u_{n}\right)=I\left(u_{\infty}\right)=V_{0},
$$

which completes the proof of the proposition in view of (6.7). Note that (6.11) implies that $u_{\infty}$ is an optimal control for the long-run average cost control problem.

The following proposition includes the second part of Theorem 6.1.

Proposition 6.3. Under the conditions of Theorem 6.1, we have

$$
\lim _{T \rightarrow \infty} \frac{V(x, T)}{T}=V_{0} .
$$

Proof. It follows from (2.5) and (2.10) that, for any $x \geq 0$ and $u>0$, we have

$$
\limsup _{T \rightarrow \infty} \frac{I(u, x, T)}{T}=I(u),
$$

where $I(u)$ is given in (3.21). Therefore

$$
\limsup _{T \rightarrow \infty} \frac{V(x, T)}{T} \leq \limsup _{T \rightarrow \infty} \frac{I(u, x, T)}{T}=I(u),
$$

and minimizing the right-hand side over $u>0$, we obtain

$$
\limsup _{T \rightarrow \infty} \frac{V(x, T)}{T} \leq \inf _{u>0} I(u)=V_{0} .
$$

It remains to show that

$$
\liminf _{T \rightarrow \infty} \frac{V(x, T)}{T} \geq V_{0}
$$

The proof of (6.13) given below is quite similar to that of (6.6). Consider a sequence of positive real numbers $\left(T_{n}\right)_{n \in \mathbb{N}}$ such that $\lim _{n \rightarrow \infty} T_{n}=+\infty$ and

$$
\liminf _{T \rightarrow \infty} \frac{V(x, T)}{T}=\lim _{n \rightarrow \infty} \frac{V\left(x, T_{n}\right)}{T_{n}} .
$$

Fix any $\varepsilon_{0}>0$, and, for each $n \in \mathbb{N}$, choose $u_{n}>0$ such that $V\left(x, T_{n}\right)+\varepsilon_{0}>I\left(u_{n}, x, T_{n}\right)$. Then, in view of (2.10) and (6.12), we have

$$
\limsup _{n \rightarrow \infty} h\left(u_{n}\right) \leq \limsup _{n \rightarrow \infty} \frac{V\left(x, T_{n}\right)}{T_{n}} \leq V_{0}<+\infty .
$$


Since $\lim _{x \rightarrow \infty} h(x)=+\infty$, this implies that $u_{n}$ is a bounded sequence. That is, there is an $M>0$ such that $u_{n} \in(0, M)$ for all $n \in \mathbb{N}$. Taking a further subsequence if necessary, we can assume without loss of generality that $u_{n}$ converges to some $u_{\infty} \in[0, M]$ as $n \rightarrow \infty$. Furthermore, $u_{\infty}>0$ because if $u_{\infty}=0$ then by (3.20) we obtain

$$
V_{0} \geq \limsup _{n \rightarrow \infty} \frac{V\left(x, T_{n}\right)}{T_{n}} \geq \limsup _{n \rightarrow \infty} \frac{I\left(u_{n}, x, T_{n}\right)}{T_{n}} \geq \mathrm{E}\left(C\left(Z_{\delta}\right)\right) \quad \text { for any } \delta>0 .
$$

This is impossible in view of part (iii) of Lemma 3.2. Therefore $u_{\infty}>0$. Let $v_{1}$ and $v_{2}$ be any numbers such that $0<v_{1}<u_{\infty}<v_{2}$. Then, by (2.10) and an argument similar to that used to derive (6.10), we have

$$
V\left(x, T_{n}\right)+\varepsilon_{0}>I\left(u_{n}, x, T_{n}\right) \geq h\left(v_{1}\right) T_{n}+\mathrm{E}\left(L_{v_{1}}^{x}\left(T_{n}\right)\right)+\int_{0}^{T_{n}} \mathrm{E}\left[C\left(X_{v_{2}}^{x}(t)\right)\right] \mathrm{d} t
$$

for large enough $n$. Using Lemma 3.1 along with (3.20), we deduce that

$$
\liminf _{n \rightarrow \infty} \frac{V\left(x, T_{n}\right)}{T_{n}} \geq h\left(v_{1}\right)+p v_{1}+\mathrm{E}\left(C\left(Z_{v_{2}}\right)\right) .
$$

Since the functions $h(u)$ and $G(u)=\mathrm{E}\left(C\left(Z_{u}\right)\right)$ are continuous, this implies that

$$
V_{0} \geq \liminf _{n \rightarrow \infty} \frac{V\left(x, T_{n}\right)}{T_{n}} \geq h\left(u_{\infty}\right)+p u_{\infty}+\mathrm{E}\left(C\left(Z_{u_{\infty}}\right)\right)=I\left(u_{\infty}\right) \geq V_{0} .
$$

The proof of (6.13) and, hence, the proof of the proposition is complete. In fact, the above inequality also shows that $u_{\infty}$ is an optimal control for the long-run average cost control problem.

Propositions 6.2 and 6.3 combined together yield Theorem 6.1.

Remark 6.1. The proofs of Propositions 6.2 and 6.3 also imply the following results.

1. Let $\left(\alpha_{n}\right)_{n \geq 0}$ be a sequence of positive numbers converging to zero, and let $u_{n}$ be an $\varepsilon$-optimal control for $V_{\alpha_{n}}(x)$ in (2.8). Then the sequence $\left(u_{n}\right)_{n \geq 0}$ is bounded and any limit point of $\left(u_{n}\right)_{n \geq 0}$ is an optimal control for $V_{0}$ defined in (2.6).

2. Let $\left(T_{n}\right)_{n \geq 0}$ be a sequence of positive numbers such that $\lim _{n \rightarrow \infty} T_{n}=+\infty$. If $u_{n}$ be an $\varepsilon$-optimal control for $V\left(x, T_{n}\right)$ in (2.9), then the sequence $\left(u_{n}\right)_{n \geq 0}$ is bounded and any limit point of $\left(u_{n}\right)_{n \geq 0}$ is an optimal control for $V_{0}$ defined in (2.6).

\section{Acknowledgements}

The work of Arka Ghosh was partially supported by the National Science Foundation grant DMS-0608634. The work of Ananda Weerasinghe was partially supported by the US Army Research Office grant W911NF0710424. We are grateful for their support.

\section{References}

[1] Ata, B., Harrison, J. M. And Shepp, L. A. (2005). Drift rate control of a Brownian processing system. Ann. Appl. Prob. 15, 1145-1160.

[2] Bell, S. L. And Williams, R. J. (2001). Dynamic scheduling of a system with two parallel servers in heavy traffic with resource pooling: asymptotic optimality of a threshold policy. Ann. Appl. Prob. 11, 608-649. 
[3] Biagini, F., Hu, Y., Oksendal, B. And Sulem, A. (2002). A stochastic maximum principle for processes driven by fractional Brownian motion. Stoch. Process. Appl. 100, 233-253.

[4] Biagini, F., Hu, Y., Oksendal, B. And Zhang, T. (2008). Stochastic Calculus for Fractional Brownian Motion and Applications. Springer, London.

[5] Budhiraja, A. And GHosh, A. P. (2005). A large deviations approach to asymptotically optimal control of crisscross network in heavy traffic. Ann. Appl. Prob. 15, 1887-1935.

[6] Budhiraja, A. And Ghosh, A. P. (2006). Diffusion approximations for controlled stochastic networks: an asymptotic bound for the value function. Ann. Appl. Prob. 16, 1962-2006.

[7] Duffield, N. G. AND O'Connell, N. (1995). Large deviations and overflow probabilities for the general single-server queue, with applications. Math. Proc. Camb. Phil. Soc. 118, 363-374.

[8] Duncan, T. E. (2007). Some stochastic systems with a fractional Brownian motion and applications to control. In Proc. American Control Conference (New York, July 2007), pp. 1110-1114.

[9] Ghosh, A. P. and Weerasinghe, A. (2008). Optimal buffer size and dynamic rate control for a queueing network with impatient customers in heavy traffic. Submitted. Available at http://www.public.iastate.edu/ apghosh/reneging_queue.pdf.

[10] Gong, W.-B. LiU, Y., Misra, V., And Towsley, D. (2005). Self-similarity and long range dependence on the Internet: a second look at the evidence, origins and implications. Comput. Networks 48, 377-399.

[11] HaIrer, M. (2005). Ergodicity of stochastic differential equations driven by fractional Brownian motion. Ann. Prob. 33, 703-758.

[12] Harrison, J. M. (1985). Brownian Motion and Stochastic Flow Systems. John Wiley, New York.

[13] Harrison, J. M. (2003). A broader view of Brownian networks. Ann. Appl. Prob. 13, 119-1150.

[14] Heath, D., Resnick, S. and Samorodnitsky, G. (1997). Patterns of buffer overflow in a class of queues with long memory in the input stream. Ann. Appl. Prob. 7, 1021-1057.

[15] Heath, D., Resnick, S. And Samorodnitsky, G. (1998). Heavy tails and long range dependence in ON/OFF processes and associated fluid models. Math. Oper. Res. 23, 145-165.

[16] Hu, Y. And Zhou, X.-Y. (2005). Stochastic control for linear systems driven by fractional noises. SIAM J. Control Optimization 43, 2245-2277.

[17] Kleptsyna, M. L., Le Breton, A. And Viot, M. (2003). About the linear-quadratic regulator problem under a fractional Brownian perturbation. ESAIM Prob. Statist. 7, 161-170.

[18] Konstantopoulos, T. and Last, G. (2000). On the dynamics and performance of stochastic fluid systems. J. Appl. Prob. 37, 652-667.

[19] Konstantopoulos, T. And Lin, S.-J. (1996). Fractional Brownian approximations of queueing networks. In Stochastic Networks (Lecture Notes Statist. 117), Springer, New York, pp. 257-273.

[20] Konstantopoulos, T., Zazanis, M. and De Veciana, G. (1996). Conservation laws and reflection mappings with an application to multi-class mean value analysis for stochastic fluid queues. Stoch. Process. Appl. 65, 139-146.

[21] Kruk, L., Lehoczky, J., Ramanan, K. and Shreve, S. (2007). An explicit formula for the Skorohod map on $[0, a]$. Ann. Prob. 35, 1740-1768.

[22] Leland, W. E., Taqqu, M. S., Willinger, W. and Wilson, D. V. (1994). On the self-similar nature of Ethernet traffic (extended version). IEEE/ACM Trans. Networking 2, 1-15.

[23] Mandelbrot, B. B. And Van Ness, J. W. (1968). Fractional Brownian motions, fractional noises and applications. SIAM Rev. 10, 422-437.

[24] Norros, I. (1994). A storage model with self-similar input. Queueing Systems 16, 387-396.

[25] Nualart, D. (2006). The Malliavin Calculus and Related Topics, 2nd edn. Springer, Berlin.

[26] Paxson, V. and Floyd, S. (1995). Wide-area traffic: the failure of Poisson modeling. IEEE/ACM Trans. Networking 3, 226-244.

[27] Sahinoglu, Z. and TeKinay, S. (1999). Self-similar traffic and network performance. IEEE Commun. Mag. $37,48-52$.

[28] Samorodnitsky, G. and Taqqu, M. S. (1994). Stable Non-Gaussian Random Processes. Chapman and Hall, New York.

[29] Shiryaev, A. N. (1999). Essentials of Stochastic Finance. World Scientic, River Edge, NJ.

[30] Taqqu, M., Willinger, W. and Sherman, R. (1997). Proof of a fundamental result in self-similar traffic modeling. Comput. Commun. Rev. 27, 5-23.

[31] Taqqu, M. S., Willinger, W., Sherman, R. and Wilson, D. V. (1997). Self-similarity through highvariability: statistical analysis of Ethernet LAN traffic at the source level. IEEE ACM Trans. Networking 5, 71-86.

[32] Weerasinghe, A. (2005). An Abelian limit approach to a singular ergodic control problem. SIAM J. Control Optimization 44, 714-737.

[33] Whitт, W. (2000). An overview of Brownian and non-Brownian FCLTs for the single-server queue. Queueing Systems 36, 39-70. 
[34] Whitt, W. (2002). Stochastic-Process Limits. Springer, New York.

[35] Willinger, W., Paxson, V. and TaqQu, M. S. (1998). Self-similarity and heavy tails: structural modeling of network traffic. In A Practical Guide to Heavy Tails: Statistical Techniques and Applications, eds R. Adler, R. Feldman, and M. S. Taqqu, Birkhauser, Boston, MA, pp. 27-53.

[36] Willinger, W., Taqqu, M. S. and Erramilli, A. (1996). A bibliographical guide to self-similar traffic and performance modeling for modern high-speed networks. In Stochastic Networks: Theory and Applications (R. Statist. Soc. Lecture Notes Ser. 4), Oxford University Press, pp. 339-366.

[37] Zeevi, A. J. AND Glynn, P. W. (2000). On the maximum workload of a queue fed by fractional Brownian motion. Ann. Appl. Prob. 10, 1084-1099. 